\title{
Análisis de áreas de actividad en el Grupo Guzmán de El Palmar, Campeche, México
}

\section{Activity Areas Analysis in the Guzman Group of El Palmar, Campeche, Mexico}

\author{
Xanti Sirani Ceballos Pesina \\ Escuela Nacional de Antropología e Historia, México \\ Agustín Ortiz Butrón \\ Luis Barba Pingarrón \\ Instituto de Investigaciones Antropológicas, \\ Universidad Nacional Autónoma de México, México \\ Araceli VázQuez Villegas \\ Escuela Nacional de Antropología e Historia, México \\ Kenichiro TSUKAmoto \\ Universidad de California, Riverside, Estados Unidos
}

\begin{abstract}
Resumen: El análisis de residuos químicos en pisos, tiene como propósito identificar las áreas de actividad en estructuras arqueológicas, particularmente en aquellas donde el abandono fue gradual y los artefactos sobre los pisos fueron escasos. Se basa en el principio de que toda actividad humana produce desechos que, al caer en los pisos en forma de líquidos, son absorbidos en sus poros y pueden ser detectados mediante pruebas de laboratorio, aún cientos de años después. Los análisis se realizaron en dos estructuras completas y dos parciales del Grupo Guzmán, una plazuela ocupada por un grupo de élite - conocido como los lakamob, "abanderados"-, ubicada a $1.3 \mathrm{~km}$ al norte del Grupo Principal de El Palmar, en Campeche. Los resultados del análisis de residuos químicos, en conjunto con el de los materiales arqueológicos y la configuración arquitectónica, permitieron identificar diversas actividades realizadas por los lakamob dentro de las estructuras, como almacenamiento, servicio y consumo de alimentos, así como otras de carácter ritual y de reunión.
\end{abstract}

Palabras Clave: El Palmar; sociedad maya clásica; lakamob; áreas de actividad; residuos químicos. 
Aвstract: The chemical residue analysis is a powerful method to identify specific activity areas in archaeological structures, like those that took place on the plaster floor of buildings, which were gradually abandoned without leaving artifacts. It is based on the principle that all human activity produces residues that, when falling on the floors in the form of liquids, are absorbed into its pores and can be detected by laboratory tests, even hundreds of years later. We analyzed four structures of the Guzmán Group, a plazuela group of the El Palmar archaeological site, which was a residential compound of non-royal elites who held the title of lakam. The results, together with other material evidence, suggest that lakamob (i.e., standard bearers) were engaged in a wide variety of activities that included food storage, consumption and serving, as well as feasting, meeting, and ritual.

KeYwords: El Palmar; Classic Maya society; lakamob; activity areas; chemical residues.

RECEPCIÓN: 24 de octubre de 2019.

ACEPTACIÓN: 19 de febrero de 2020.

Dor: https://doi.org/10.19130/iifl.ecm.57.2021.18653

\section{Introducción}

La aplicación de los análisis de fosfatos en el campo de la agronomía fue descubierta por O. Arrhenius en la década de 1930 y, a partir de entonces, los arqueólogos comenzaron a utilizarla como una herramienta para detectar asentamientos humanos (Mejía y Barba, 1988: 129). En México, la primera vez que se detectó la relación entre las actividades humanas y el enriquecimiento químico del suelo mediante la distribución de fosfatos fue en Xochipala, Guerrero, con un estudio realizado por Luis Barba (1990: 271); debido a éste y otros estudios etnográficos y arqueológicos (Barba y Bello, 1978), se concluyó que las actividades humanas, tales como la preparación de alimentos, el comer y el realizar ofrendas producen desechos que pueden ser detectados mediante pruebas sencillas de laboratorio, aunque hayan transcurrido muchos años (Barba, 1986: 22). Posteriormente, el trabajo sobre áreas de actividad mesoamericanas, llevado a cabo por Luis Barba y Linda Manzanilla, se basó en la premisa de que las unidades habitacionales son grupos de actividad — tal como la definen Ashmore y Wilk (1988)—; por lo tanto, el estudio de dichas actividades mediante los análisis de residuos permite entender cómo funcionaban los espacios en la antiguiedad (Manzanilla y Barba, 1990: 41).

Las actividades de un grupo destacan al ser comparadas con las actividades de otros grupos sociales; por ello, para definir la función de las estructuras del Grupo Guzmán del sitio El Palmar, en Campeche, fue necesario hacer una comparación entre ellas. El análisis de residuos químicos, tales como fosfatos, carbonatos, ácidos grasos, proteínas, $\mathrm{pH}$ y carbohidratos, junto con el análisis de la distribución de los artefactos recuperados en excavación y la organización espacial a través de la arquitectura, proporcionaron un panorama más amplio de 
dicho conjunto y de la dinámica social de los lakamob, un grupo de la élite no-real en la sociedad maya clásica (600-850 d.C.) que lo habitó.

Los análisis de residuos se realizaron en el Laboratorio de Prospección Arqueológica del Instituto de investigaciones Antropológicas de la Universidad Nacional Autónoma de México. El total de muestras de piso fue de 163 y el análisis se llevó a cabo en tres etapas: en el año 2017 fueron procesadas 34; en el 2018 se analizaron 120, y en el año 2019 se procesaron 9 más. Las muestras de 2017 incluyeron 14 de la Estructura GZ1 y 20 del cuarto central de la Estructura GZ6; las muestras de piso del 2018 y 2019 se divididen en 53 de la Estructura GZ3 y 76 de la Estructura GZ5, esto con el fin de identificar las áreas de actividad y comparar los resultados entre ellas.

\section{El sitio de El Palmar, Campeche, México}

El Palmar se encuentra al sureste del estado de Campeche, México, a $50 \mathrm{~km}$ al oriente de Calakmul (Figura 1). Desde el año 2007 a la actualidad, se ha desarrollado allí el Proyecto Arqueológico El Palmar: urbanización del espacio social en las tierras bajas mayas, codirigido por Kenichiro Tsukamoto y Javier López Camacho.

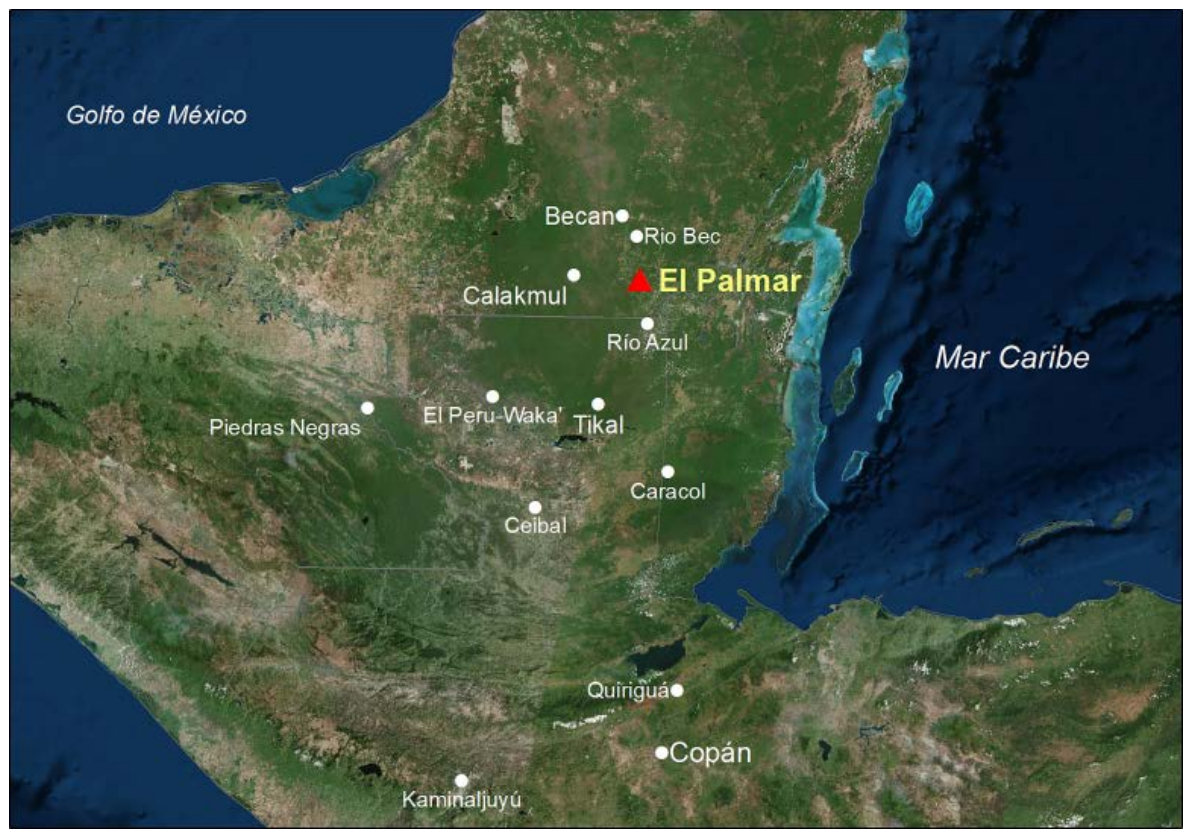

Figura 1. Mapa de ubicación de El Palmar y sitios aledaños

(Según Google Maps, modificado por Kenichiro Tsukamoto). 
Durante la primera temporada de campo, el proyecto estuvo enfocado en el reconocimiento y la excavación de pozos en el Grupo Principal; sin embargo, el objetivo de la investigación se modificó en el año 2009, al descubrirse bloques con inscripciones jeroglíficas en el Grupo Guzmán (Figura 2), localizado a $1.3 \mathrm{~km}$ al norte del Grupo Principal, el cual está compuesto por una estructura tipo templo al este y siete estructuras alargadas alrededor de una plaza, denominado como patrón de Plaza Plan 2 (Becker, 1991).

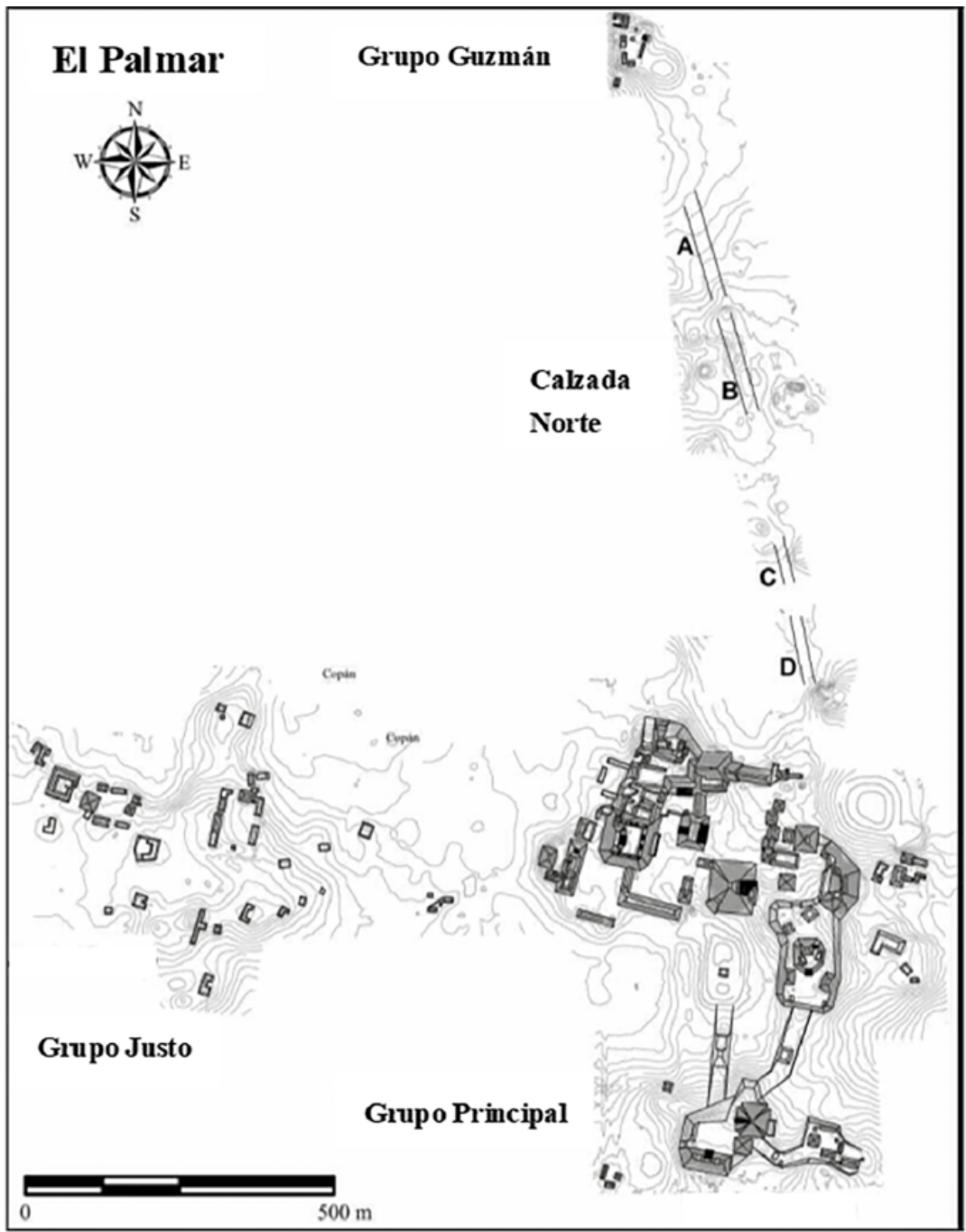

Figura 2. Plano de El Palmar, donde se observa el Grupo Principal y el Grupo Guzmán al norte (Mapa y dibujo: Kenichiro Tsukamoto y Javier López Camacho). 


\section{Intervención arqueológica en el Grupo Guzmán}

Durante las temporadas de campo 2010-2016, se realizaron las intervenciones arqueológicas de varias estructuras en el Grupo Guzmán. Debido a la importancia de preservar y documentar el hallazgo de la escalinata jeroglífica, en los años 2010-2011 se iniciaron los trabajos de excavación, que dejaron al descubierto un total de lo que se estima fueron 164 bloques con inscripciones, distribuidos en seis escalones, adosados a una estructura cuadrangular con un templo (Tsukamoto y Esparza, 2016: 33).

La escalera jeroglífica da cuenta del grupo social lakam, cuyo logograma fue identificado por David Stuart (2010), quien lo tradujo como "bandera” en maya yucateco; posteriormente, Alfonso Lacadena (2008) sugirió que lakam o "abanderado" fue el título de un segmento menor en la sociedad maya, dedicado a la administración del tributo y las labores militares ya que, hasta ese momento, no se había encontrado mención de ellos en registros epigráficos monumentales. La creación de la plaza, contemporánea a la escalera jeroglífica, y el incremento en el ancho de los peldaños han sido interpretados como un acondicionamiento realizado por los lakamob, quienes fueron un grupo de la élite no-real, con el fin de que la audiencia observara las representaciones teatrales llevadas a cabo en la parte superior del templo (Tsukamoto, 2014: 63).

Durante las temporadas de campo 2010-11 y 2012 se llevó a cabo la excavación de la Estructura GZ6 con el fin de conocer las actividades cotidianas realizadas en el Grupo Guzmán (Tsukamoto et al., 2015). La estructura consiste en tres cuartos: el Cuarto 1, que es el más grande, se ubica al poniente y cuenta con una banqueta lateral y una banqueta central; el Cuarto 2, ubicado al centro,

y el Cuarto 3, en el extremo oriente con una banqueta, que presenta orientación oriente-poniente (Figura 3).

En la temporada de campo 2016 se excavaron las estructuras GZ3 y GZ5, con el fin de ampliar la información que se tenía hasta la fecha. La Estructura GZ3 consta de dos cuartos con orientación oriente-poniente, cada uno con una banqueta, entrada independiente y conexión a la parte sur a través de una puerta en el cuarto poniente; mientras que la Estructura GZ5 es una galería corrida de norte a sur, con cinco vanos y una banqueta al poniente, sin puerta que conecte con ella (Tsukamoto et al., 2017).

Tanto en la Estructura GZ1 como en GZ6 se registraron rituales de terminación, con abundante material cerámico y lítico a nivel de piso, mientras que en GZ3 se documentó un ritual de terminación con un ensamble más pequeño y en GZ5 un ritual compuesto en su mayoría por lítica. El análisis tipo-variedad de la cerámica y las dataciones proporcionadas por el análisis de Carbono 14, demuestran que estos materiales fueron depositados sobre el piso alrededor del año 850 d.C., antes del abandono del Grupo Guzmán (Tsukamoto, 2017; Ceballos, 2020). Las cuatro estructuras mencionadas son el objeto de estudio del presente trabajo. 


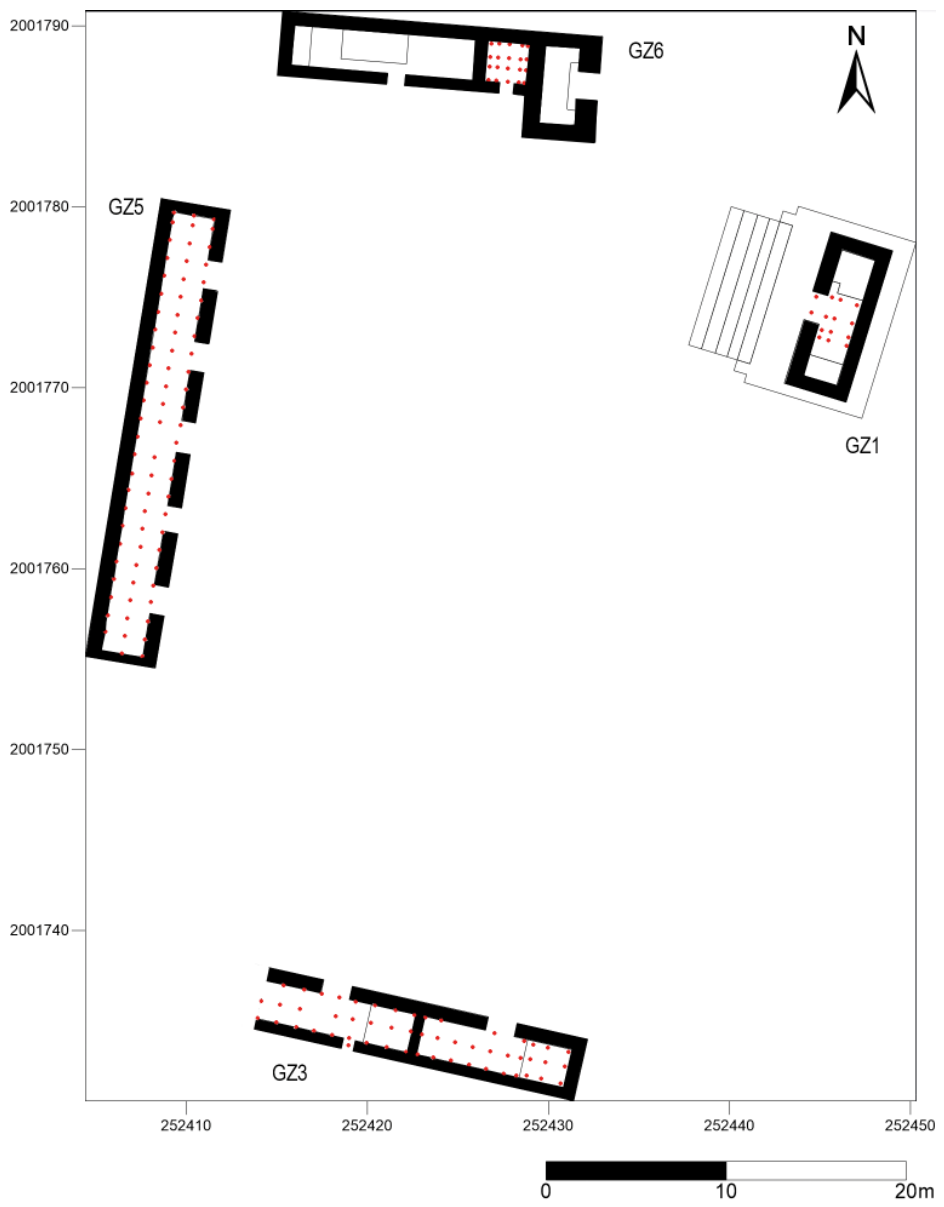

Figura 3. Plano de distribución de puntos de muestreo en las estructuras del Grupo Guzmán (Dibujo: Kenichiro Tsukamoto y Xanti Ceballos).

\section{Métodología de campo}

Las muestras de piso correspondientes a las Estructuras GZ6 y GZ1 fueron tomadas parcialmente durante la temporada de campo 2010-2011, con el fin de usarse en análisis químicos posteriores. Al tratarse de espacios más pequeños, ambos cuartos se dividieron en una cuadrícula de $0.50 \times 0.50 \mathrm{~m}$, de donde se extrajeron bloques de muestra que se almacenaron en la Escuela Nacional de Antropología e Historia (Tsukamoto y López Camacho, 2011: 149).

Por otro lado, el muestreo de las Estructuras GZ3 y GZ5 se llevó a cabo durante las temporadas de campo 2018 y 2019. Con ayuda de una cinta métrica y 
flexómetros, se trazó una cuadrícula a lo largo y ancho de las crujías de ambas estructuras; los lugares donde se hicieron sondeos en temporadas anteriores ya habían sido tomadas y fueron evitadas. El ancho de la galería interior de ambas estructuras es de $2.1 \mathrm{~m}$, por lo que, para cubrir completamente la superficie, se dividió el ancho entre tres líneas: la primera se colocó a $0 \mathrm{~m}$, la segunda a 1.05 $\mathrm{m}$ y la tercera a $2.1 \mathrm{~m}$, teniendo como resultados cuadros de $1.05 \mathrm{~m}$ de ancho $\mathrm{x}$ $1 \mathrm{~m}$ de largo. Se consideró importante tomar las muestras cerca de las orillas, ya que estudios etnográficos y arqueológicos anteriores han encontrado la mayor concentración de residuos en las esquinas de las estructuras y los patios asociados; esto se debe a que las mismas no se limpian a profundidad, además de ser los puntos donde se acumula el descarte provisional, lo que se refleja en una mayor concentración de residuos en esas áreas específicas (Hayden y Cannon, 1983: 131; Hutson et al., 2007: 457).

En 2016, al término de las labores de consolidación en el Grupo Guzmán, la superficie del piso se cubrió con sascab fino para protegerlo, lo cual de ninguna manera altera o difumina los cambios en las propiedades del suelo ocasionadas por las actividades que ahí se practicaron (Barba y Manzanilla, 1987: 107). Por lo tanto, en 2018, con el fin de optimizar el tiempo en la toma de muestras, se utilizó un martillo y una tabla para golpear un tubo de $\mathrm{PvC}^{1} \mathrm{de} 10 \mathrm{~cm}$ de diámetro, apoyado sobre la tierra cribada que se le había puesto para proteger los pisos, con el fin de extraer un bloque de piso que quedase atorado en el tubo - a modo de barreno- y raspar el contenido dentro de una bolsa libre de fosfatos, previamente marcada con el alfanumérico del cuadrante correspondiente. La cucharilla se lavó y secó entre cada una de las muestras. Posteriormente las coordenadas UTM $^{2}$ de las muestras se recuperaron en la versión georreferenciada del AutoCAD, donde las plantas de las estructuras están trazadas con precisión por una estación total Leica TS06 plus (Figura 3).

La Estructura GZ3 se muestreó en toda su extensión incluyendo la banqueta del cuarto oriente, donde se observó el recubrimiento de estuco totalmente degradado, mientras que la banqueta poniente de la Estructura GZ5 no pudo ser muestreada debido a que, en la temporada anterior, su superficie fue consolidada con lajas de piedra (Tsukamoto y López Camacho, 2018: 29).

\section{Metodología en el laboratorio}

El método a seguir para determinar la presencia o ausencia de los residuos químicos en pisos y cerámica arqueológicos es el descrito en el Manual de técnicas microquímicas de campo para la arqueología (Barba, Rodríguez y Córdova, 1991), al que se han añadido mejoras a través de los años (Barba, 2007). Las muestras

\footnotetext{
${ }^{1}$ Policloruro de vinilo, un tipo de plástico rígido.

2 Sistema de coordenadas internacional "Universal Transversa de Mercator", el cual proporciona las medidas de $x$ (este), $y$ (norte) y $z$ (altura) en metros.
} 
recolectadas fueron sometidas a seis pruebas, las cuales ofrecen diferente información: los fosfatos detectan residuos de tejidos orgánicos y son el indicador más antiguo y común para detectar actividades humanas; los carbonatos son indicadores de las características del piso y ofrecen información sobre su conservación; los carbohidratos indican la presencia de sustancias ricas en almidones y azúcares; los ácidos grasos muestran el lugar donde hubo impregnación de grasas humanas, aceites o resinas de origen animal o vegetal; el pH es un indicador de áreas de combustión; por último, las proteínas se encuentran en tejidos y fluidos de humanos y animales.

Además de lo que indica cada prueba, hay consideraciones que se deben tener en cuenta al interpretar los resultados. Por ejemplo, al estudiar el área maya, no debe sorprender que los análisis de carbonatos resulten especialmente altos, ya que, como mencionan Barba et al. (1997: 50), su distribución está directamente relacionada con el material empleado en la construcción del piso, y en el área maya la cal es determinante en la concentración de carbonatos. También han notado que el deterioro de la superficie modifica la proporción y está directamente relacionado con la conservación del piso: mientras mejor conservado se encuentre, mayor enriquecimiento de carbonatos presentará. Asimismo, en estudios anteriores se ha relacionado el $\mathrm{pH}$ alcalino con áreas donde se encontraban fogones y, en general, con evidencias de combustión (Manzanilla y Barba, 1990: 42 ); sin embargo, en el área maya, se debe tener en cuenta que un $\mathrm{pH}$ alto es característico de los pisos estucados, por la alcalinidad de los carbonatos, además de que un valor mayor a 9 puede indicar la presencia de cenizas.

\section{Resultados del análisis químico en el Grupo Guzmán}

Una vez realizadas las pruebas, se procedió a elaborar los mapas de distribución de los valores de residuos, con la finalidad de interpretar los patrones de enriquecimiento en las estructuras y, por ende, identificar las áreas de actividad humana. Los datos fueron procesados en el programa Golden Surfer 14: cada muestra recolectada se georreferenció en $x$ y $y$ con las coordenadas UTM correspondientes, mientras que los valores de $z$ fueron sustituidos por los valores de cada análisis, lo que genera un mapa de curvas de nivel que, mediante el cambio de colores, muestra patrones de enriquecimiento de residuos.

A continuación se presentan los resultados del análisis, el cual está enfocado en las Estructuras GZ3 y GZ5, mientras que las Estructuras GZ1 y GZ6 los complementan, debido a que sólo fueron muestreadas parcialmente.

\section{Estructura GZ3}

En el mapa de distribución de fosfatos (Figura 4), se puede notar que el valor más alto se presenta en las esquinas u orillas de la estructura, donde, en general, se 
concentran más los residuos, lo que concuerda con lo encontrado en los estudios etnográficos y arqueológicos mencionados. El valor de fosfatos más alto se encuentra en los pisos de ambos cuartos y en la banqueta oriente, mientras que la banqueta poniente tiene un enriquecimiento de fosfatos en concentraciones más bajas que el resto de la estructura, a excepción de tres esquinas, quizá porque las actividades realizadas en ella fueron menos intensas y/o frecuentes, lo que enriqueció poco esta área.

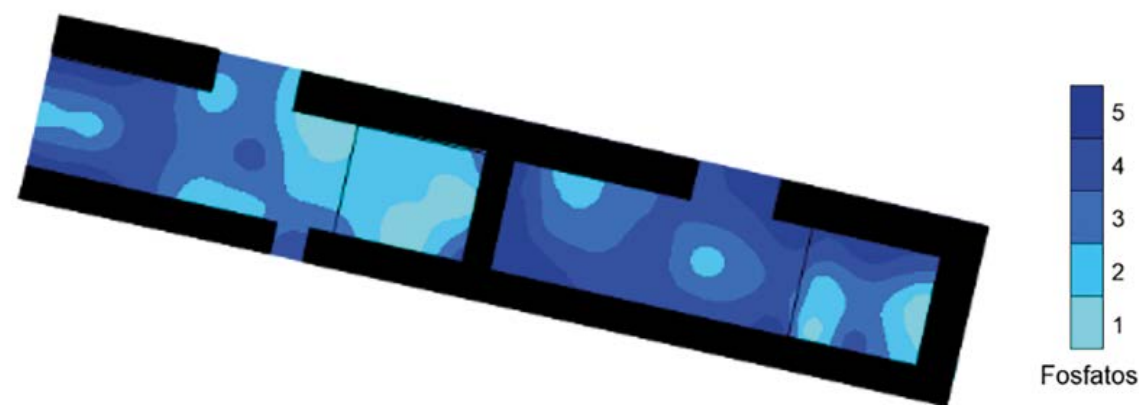

Figura 4. Distribución de fosfatos en la Estructura GZ3

(Dibujo: Xanti Ceballos y Kenichiro Tsukamoto).

En la Estructura GZ3 podemos notar una diferencia en la concentración de carbonatos entre los dos cuartos (Figura 5), ya que el del poniente tiene una distribución casi homogénea y valores ligeramente más bajos que el cuarto oriente, a excepción de las esquinas noroeste y sureste de la banqueta. En contraste, el cuarto oriente presenta valores altos, concentrados en el lado opuesto a la banqueta. En general, en las banquetas hay valores bajos de carbonatos, que pueden estar relacionados con el desgaste por el uso constante para realizar ciertas actividades, como dormir.
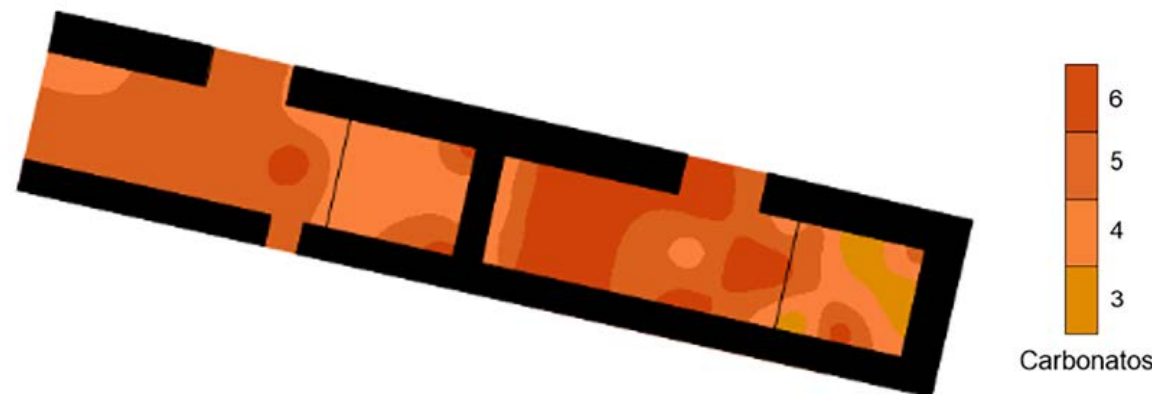

Figura 5. Distribución de carbonatos en la Estructura GZ3 (Dibujo: Xanti Ceballos y Kenichiro Tsukamoto). 
Comparando ambos cuartos, se puede afirmar que, aunque no se aprecia a simple vista, el cuarto poniente puede tener diferencias en la proporción de cal de su piso o bien se fue deteriorando a nivel químico y perdiendo componentes. Lo anterior es importante ya que, cuando la estructura fue excavada, a simple vista el piso de los cuartos y de la banqueta preservada se veían en buen estado de conservación, sin deterioros visibles (Tsukamoto y López Camacho, 2018: 59). Los fosfatos indican que el cuarto oriente está más enriquecido; sin embargo, los carbonatos muestran que el cuarto poniente tiene una concentración menor, quizá porque las actividades ahí llevadas a cabo fueron más intensivas, pero ligeramente menos contaminantes. Esto concuerda con la observación de Barba et al. (1997: 52), en el estudio de pisos en la Casa de las Águilas de Tenochtitlan, donde los carbonatos en bajas concentraciones frente al altar principal se atribuyeron al uso intensivo del área.

A diferencia de otros residuos, las proteínas se presentaron en lugares muy específicos de las estructuras (Figura 6). En GZ3 la mayor concentración de ellas se encuentra en la banqueta del cuarto oriente; en el cuarto poniente se hallan los valores más elevados en dos esquinas de la banqueta, así como en el piso a un costado de ella, mientras que en el cuarto oriente las proteínas están en la esquina suroeste del cuarto y en una pequeña sección del centro de la habitación. Esto podría corresponder a áreas donde se consumían alimentos ricos en proteínas.

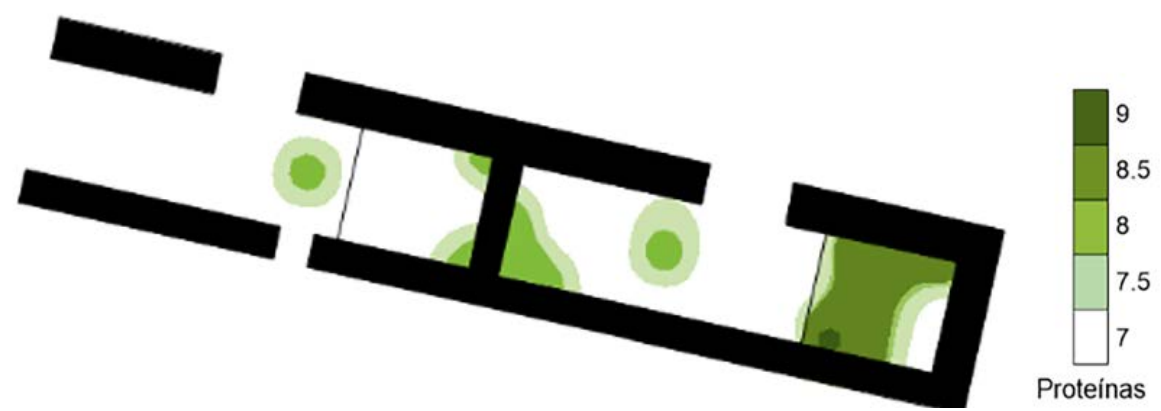

Figura 6. Distribución de proteínas en la Estructura GZ3 (Dibujo: Xanti Ceballos y Kenichiro Tsukamoto).

En la Estructura GZ3 (Figura 7), los ácidos grasos se encontraron concentrados principalmente en las banquetas, lo que puede estar asociado a las actividades que ahí se realizaban, como el consumo de alimentos, la recepción de visitas y dormir, lo que dejaría una impregnación de grasas humanas si se hacía en contacto directo con la superficie (Barba y Ortiz, 1992: 72). En ambos cuartos se pueden notar zonas dispersas con enriquecimientos de ácidos grasos y muy pocos espacios carentes de ellos. Teniendo en cuenta las dimensiones y características de la estructura se podría hablar de un espacio donde se almacenaron, prepararon y consumieron alimentos ricos en grasas vegetales o animales. En el cuarto oriente, 
las mayores concentraciones están en la esquina suroeste, cerca de donde fue encontrada una laja de pedernal de forma romboidal con una superficie lisa, que pudo ser utilizada como mortero para moler alimentos o para machacar pigmentos y arcilla (Triadan, 2000: 51).
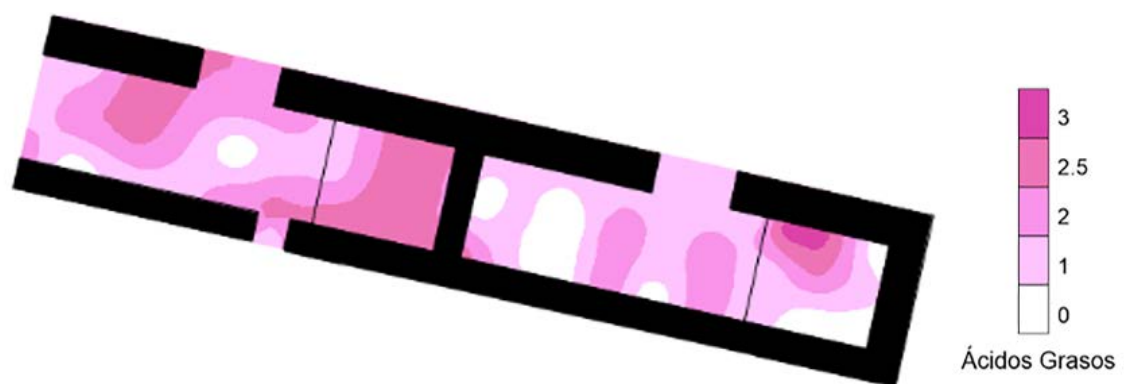

Figura 7. Distribución de ácidos grasos en la Estructura GZ3 (Dibujo: Xanti Ceballos y Kenichiro Tsukamoto).

Es interesante notar que los ácidos grasos, al igual que las proteínas, se concentran en las banquetas, lo cual reforzaría la propuesta del servicio y consumo de alimentos en esos espacios más que su preparación, ya que no se encontró evidencia alguna de un fogón dentro de la estructura, lo que sugeriría que la cocción se realizaba en otra parte, posiblemente al exterior de la misma.

De igual manera, llama la atención la abundante presencia de ácidos grasos, pues es uno de los indicadores químicos que más fácilmente se pierden en el área maya. La variación de $\mathrm{pH}$ en todas las estructuras fue mínima, con ligeras diferencias entre ellas, teniendo un promedio de 9.28, un mínimo de 8.73 y un máximo de 9.46; sin embargo, los valores más altos están en la Estructura GZ3 (Figura 8). La variación del pH no es significativa, por lo que se podría afirmar que el valor alcalino es una mezcla entre los componentes del piso y las cenizas del ritual de terminación que se documentó en todas las estructuras. Los valores bajos de $\mathrm{pH}$ en la banqueta oriente pueden deberse al mal estado de conservación del recubrimiento de estuco.

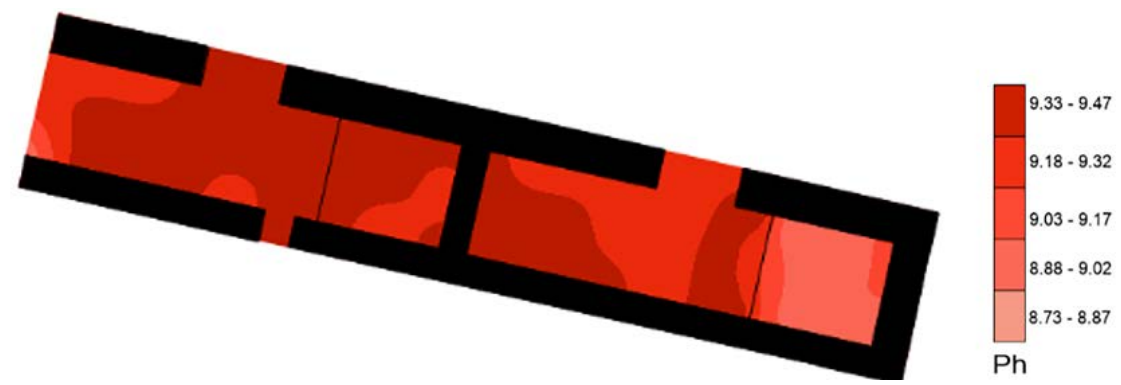

Figura 8. Distribución de pH en la Estructura GZ3 (Dibujo: Xanti Ceballos y Kenichiro Tsukamoto). 
Los valores más altos de carbohidratos se encontraron en las esquinas de los cuartos, en una esquina de la banqueta poniente y la mayor parte de la banqueta oriente. La presencia de los carbohidratos en los pisos de las estructuras es, quizá, más complejo de interpretar; sin embargo, un estudio anterior, llevado a cabo por Tsukamoto (2017: 1644), sugiere que a lo largo de los 200 años que el Grupo Guzmán fue ocupado, y tras la construcción de la Plaza A, los lakamob llevaron a cabo varios festejos donde se servían banquetes, posiblemente con el fin de negociar su estatus político y social con otros segmentos sociales; la evidencia del uso intenso de las vasijas indica que las festividades con alimentos y bebidas fueron llevadas a cabo constantemente.

Si las festividades en el Grupo Guzmán se realizaban con frecuencia e incluían a todos los habitantes, se podría pensar que esta distribución de carbohidratos en las estructuras podría deberse a un enriquecimiento asociado a bebidas rituales ricas en azúcares y almidones, derramadas ya sea como parte del ritual o por accidente como efecto de la bebida embriagante. En el caso de GZ3, los valores son bajos en toda la unidad, a excepción de las esquinas, lo que se puede asociar a un consumo moderado pero constante de tales bebidas (Figura 9).

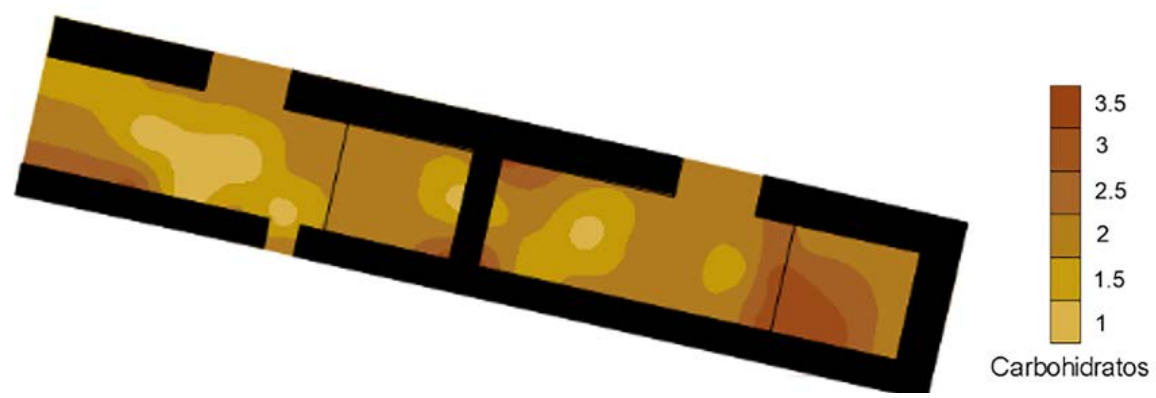

Figura 9. Distribución de carbohidratos en la Estructura GZ3 (Dibujo: Xanti Ceballos y Kenichiro Tsukamoto).

Un enriquecimiento de carbohidratos se reportó en la Casa de las Águilas, donde se asoció a la bebida fermentada de pulque (Barba et al., 1998: 25), mientras que un estudio reciente de arqueología experimental sobre la producción y venta de pulque mostró que los valores de carbohidratos se incrementaban en áreas donde se había esparcido pulque, sin mostrar incremento significativo de otros residuos (Pecci, Ortiz y Barba, 2017). Además, al realizarse una cromatografía de gases acoplada a espectrometría de masas a la misma muestra experimental, se encontraron compuestos relacionados con la fermentación, como la del vino, pero en diferentes concentraciones, lo que muestra que las bebidas fermentadas y alcohólicas pueden identificarse en el registro arqueológico (Pecci, Ortiz y Barba, 2017: 49-50). En este caso particular, al comparar el experimento con las zonas de enriquecimiento en un taller de producción de pulque tradicio- 
nal en Hidalgo, se encontró que los valores más altos de carbohidratos estaban presentes en las áreas en que se colocan los recipientes del pulque, que es donde se sirve, y donde las personas se sientan a tomar la bebida. También se observaron manchas de pulque en el piso, debido a la acción de verterlo, lo que se ve reflejado en el enriquecimiento químico (Pecci, Ortiz y Barba, 2017: 51).

Por lo tanto, se sugiere que el enriquecimiento de carbohidratos podría ser causado por el consumo de bebidas altas en carbohidratos, como líquidos a base de maíz o cacao, endulzado con miel, y/o bebidas fermentadas como el balché u otra similar que pudieron haber sido derramadas sobre el piso. David Stuart (1988: 156) menciona que hay evidencia en los glifos de vasijas "someras" de la fase Tepeu, de que fueron usadas como recipientes para beber atole, o lo que los mayas llaman $u l$, una bebida de maíz, así como de vasos cilíndricos adornados con el glifo del cacao, también del Clásico Tardío.

\section{Estructura GZ5}

En esta estructura el enriquecimiento de fosfatos es, en general, bajo en la mayor parte de la galería, mientras que los valores más altos se presentan al centro y en las esquinas de la misma (Figura 10). Sin embargo, esta primera prueba química podría indicar que la estructura no tuvo una función doméstica, ya que es inusual encontrar valores tan bajos de fosfatos en un lugar donde se desarrollaron actividades cotidianas que impregnaron los pisos de sustancias a lo largo del tiempo (Ortiz y Barba, 1992: 122).

En contraste, los niveles de carbonatos en general son altos, a excepción de algunos puntos cercanos a los accesos de la estructura; también se puede observar una ligera disminución de ellos al centro de la misma (Figura 11). De lo anterior, podemos inferir que el aumento de carbonatos indica que las actividades realizadas no fueron intensivas, lo que permitió conservar alta su proporción. Otra explicación que hay que tener en cuenta es el factor del tiempo, ya que quizá este piso fue construido en una época posterior al de las otras estructuras, lo que le impidió enriquecerse de la misma forma.

En el caso de las proteínas, se puede notar que se concentran en el sector norte de la estructura y sólo una pequeña cantidad cerca del tercer vano, de sur a norte (Figura 12). Esta distribución no es clara, debido a que los otros valores no apoyan la idea de que GZ5 haya sido destinada a preparar o consumir alimentos.

Asimismo, se observa un enriquecimiento de ácidos grasos en bajas concentraciones y en lugares específicos: al centro y en las esquinas del muro poniente, algunos detrás de las jambas y en una sección al centro (Figura 13). La distribución no parece tan aleatoria y podría deberse a que en esos lugares estuvo algún objeto con grasas animales o vegetales, o bien, resinas de madera que dejaron impregnada la superficie (Barba y Ortiz, 1992: 79), lo que se explicaría por la presencia de vigas que sostenían una techumbre de material perecedero. 

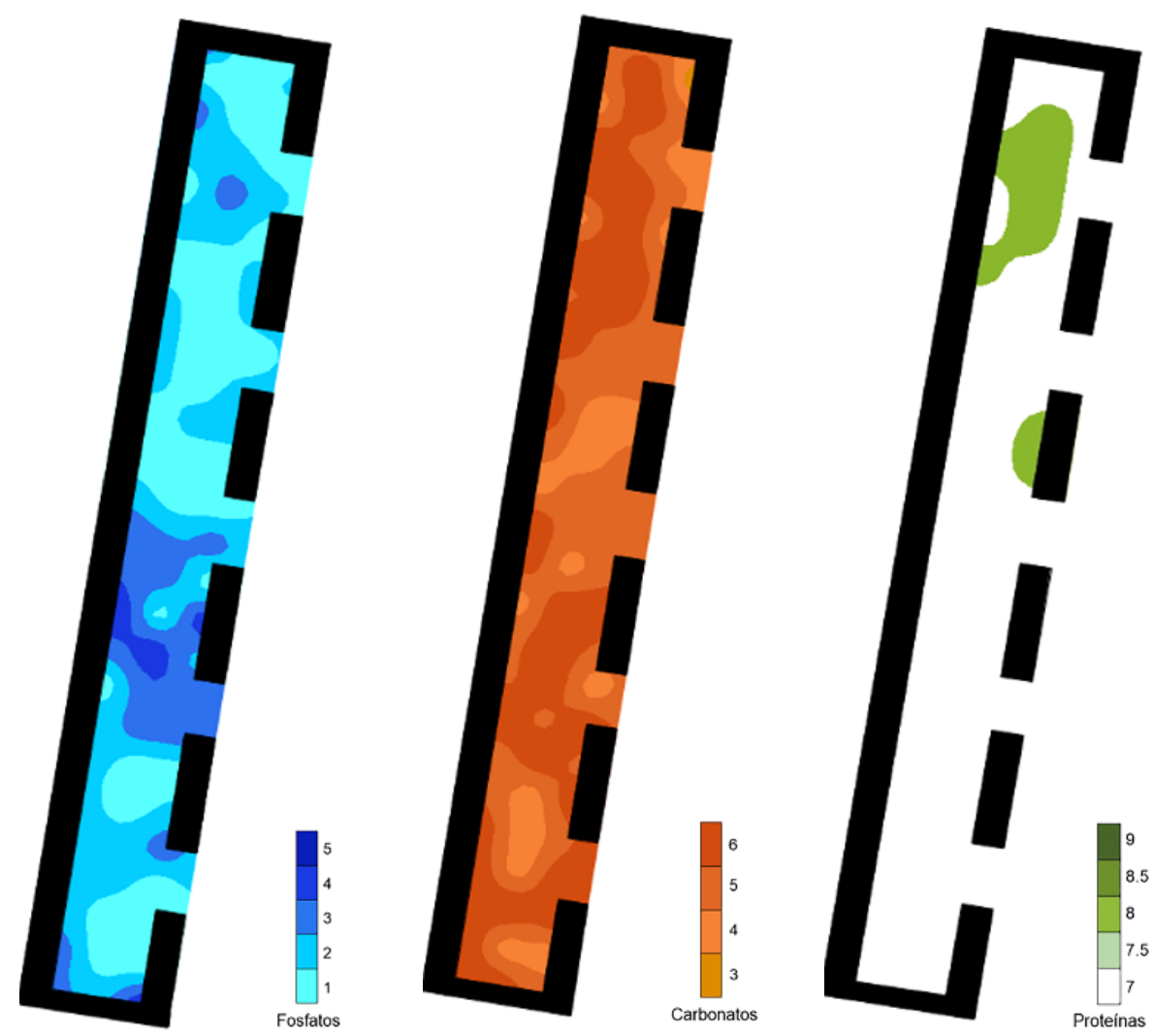

Figuras 10,11 y 12 . Distribución de fosfatos, carbonatos y proteínas en la Estructura GZ5 (Dibujo: Xanti Ceballos y Kenichiro Tsukamoto).

Los valores más altos de $\mathrm{pH}$ se encuentran en la mitad poniente; sin embargo, como ya se mencionó, no presentan cambios significativos entre ellos, y los valores más bajos pueden relacionarse al deterioro químico del piso y, por lo tanto, a la pérdida de su alcalinidad (Figura 14). En el caso de los carbohidratos (Figura 15), hay algunos valores más altos que los encontrados en GZ3, y se encuentran cerca de los vanos de la estructura, así como en la sección norte.

En esta estructura sólo se encontró un cuello de olla sobre el nivel de piso y un cuenco casi completo de $36 \mathrm{~cm}$ de diámetro; los demás tiestos del mismo contexto primario eran demasiados pequeños, sin formas completas o semicompletas; cabe mencionar que la mayoría de los artefactos encontrados sobre el piso fueron instrumentos líticos, como bifaciales ovalados, perforadores, núcleos, lascas con huellas de uso, lascas sin huellas de uso, lascas de descortezamiento y tajadores. Los materiales encontrados, en conjunto con el bajo enriquecimiento 

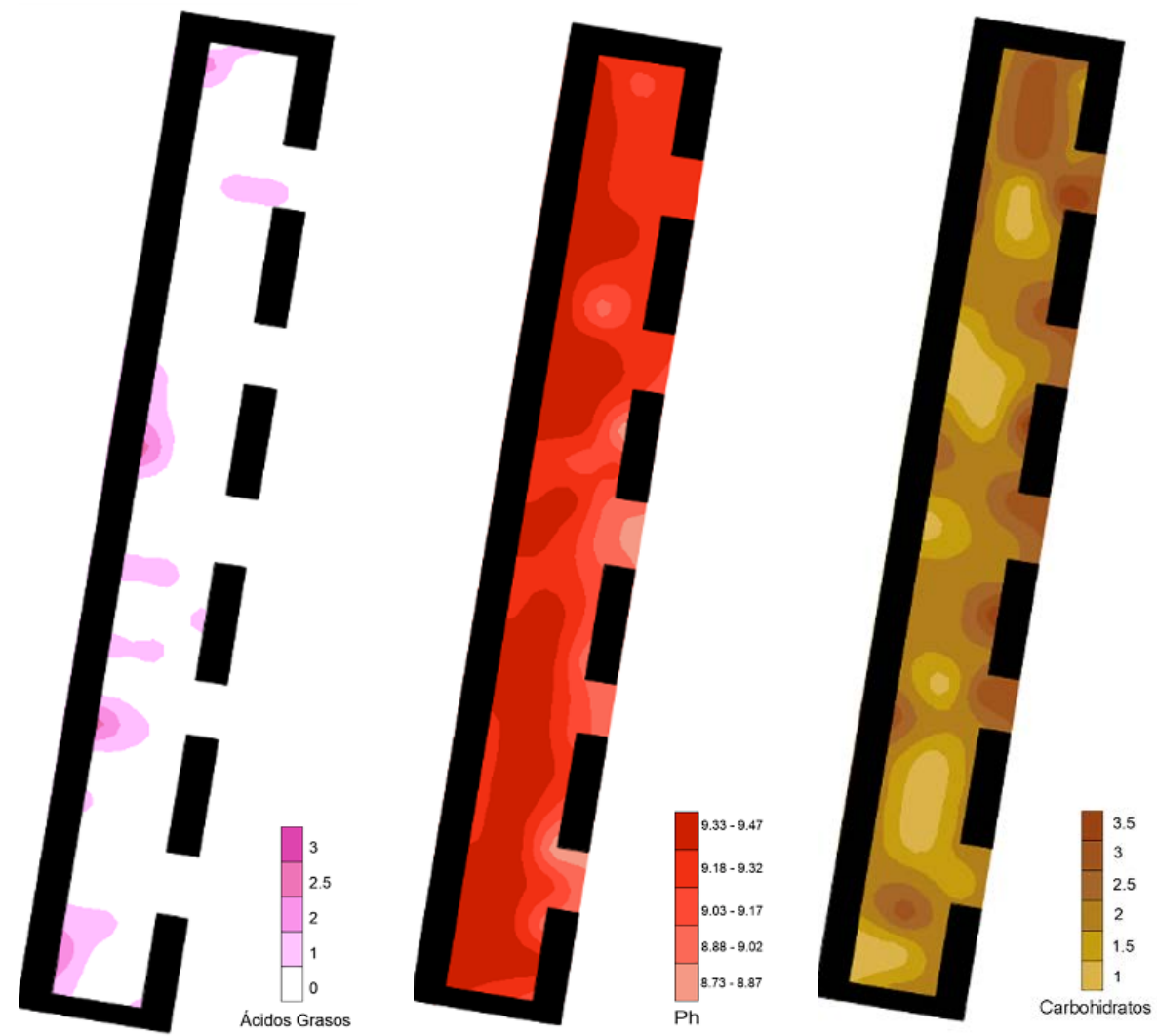

Figuras 13, 14 y 15. Distribución de ácidos grasos, pH y carbohidratos en la Estructura GZ5 (Dibujo: Xanti Ceballos y Kenichiro Tsukamoto).

del piso, pueden indicar una función administrativa, que no la excluiría de los banquetes que se llevaban a cabo en el Grupo Guzmán.

\section{Estructura GZ1}

Los niveles de fosfatos en el área muestreada son en general bajos, a excepción de la entrada al templo y la parte norte de la estructura (Figura 16); asimismo podemos notar que, al igual que GZ5, presenta un piso con altas concentraciones de carbonatos (Figura 17), lo cual se puede explicar por su función ritual como templo y por las restringidas actividades que ahí se llevaban a cabo, lo que preservó el piso.

En la Estructura GZ1, las proteínas se concentran en el vano, así como en la mitad norte de la estructura, mientras que la mitad sur prácticamente no tiene 

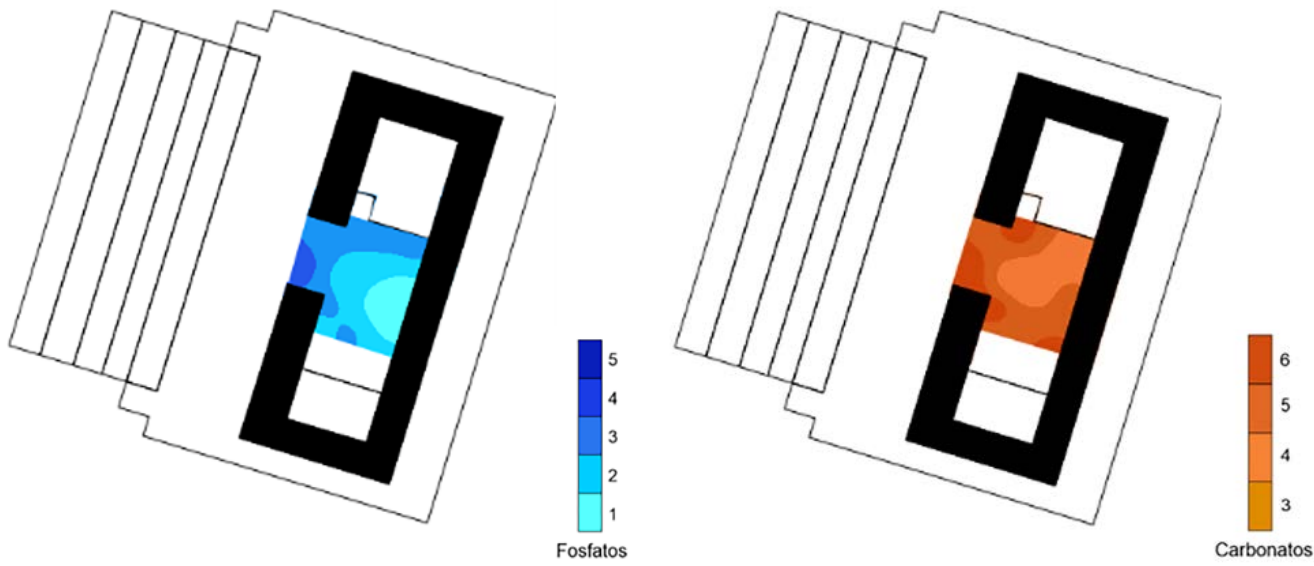

Figuras 16 y 17. Distribución de fosfatos y carbonatos en la Estructura GZ1 (Dibujos: Xanti Ceballos y Kenichiro Tsukamoto).

rastro de ellas (Figura 18). Los valores en este cuarto son más elevados que en las dos estructuras anteriores, lo cual puede estar relacionado con su función de templo, donde se habrían desarrollado actividades rituales (Tsukamoto et al., 2015: 215). En dichas actividades quizá se utilizó una ofrenda de sangre humana o animal, lo cual explicaría las altas concentraciones protéicas en esta área reducida (Barba et al., 1996: 149; Ortiz, Barba y Pecci, 2017). Aunado a lo anterior, el cuarto presenta un enriquecimiento homogéneo de ácidos grasos (Figura 19), con la mayor concentración cerca de la entrada que, al igual que las proteínas, puede estar asociado con la actividad ritual que ahí se llevó a cabo, y que pudo implicar el uso de alguna resina con ácidos grasos vegetales o alguna ofrenda con grasas animales (Barba et al., 1997).
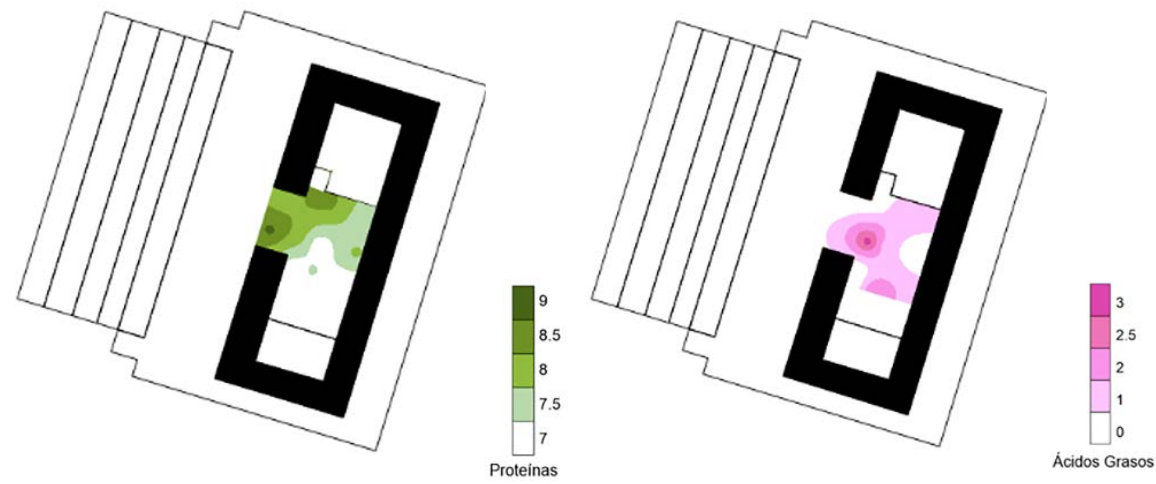

Figuras 18 y 19. Distribución de proteínas y ácidos grasos en la Estructura GZ1 (Dibujos: Xanti Ceballos y Kenichiro Tsukamoto). 
El pH tiene valores arriba de 9 y no se presentan grandes variaciones en el cuarto (Figura 20); probablemente se debe a los factores antes mencionados, así como a la presencia de cenizas que formaron parte del ritual de terminación. Los carbohidratos presentan su mayor enriquecimiento en la entrada y junto a la banqueta norte (Figura 21).
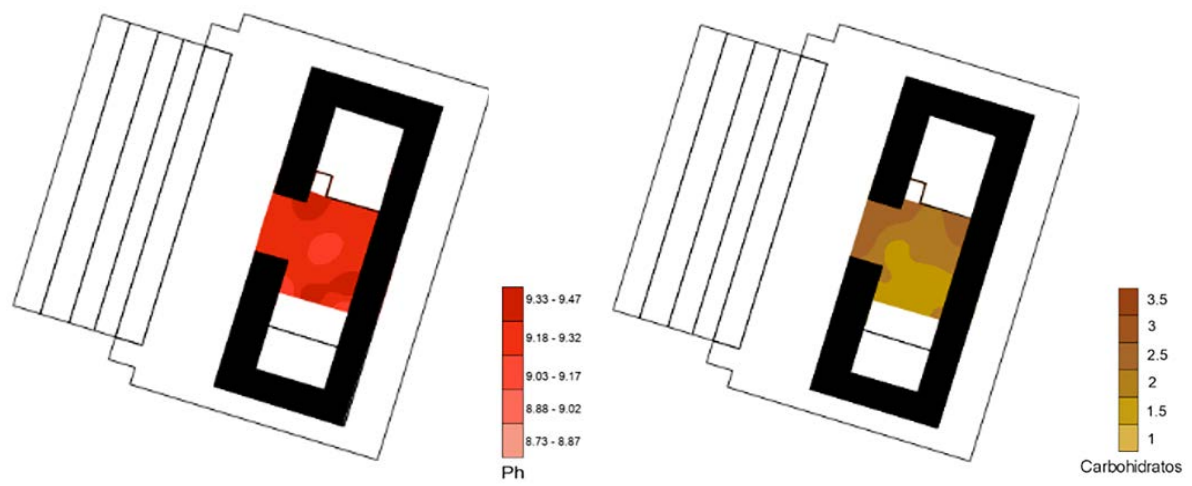

Figuras 20 y 21. Distribución del pH y carbohidratos en la Estructura GZ1 (Dibujos: Xanti Ceballos y Kenichiro Tsukamoto).

Es interesante notar que las áreas con valores altos de residuos en esta estructura están concentradas en la entrada y cerca de la banqueta norte, la cual es más grande que la banqueta sur, lo que, junto con los enriquecimientos, hablaría de su importancia ritual. La distribución de los residuos químicos, como ácidos grasos y proteínas, se puede relacionar con la función ritual de GZ1, así como la escena iconográfica plasmada en un vaso cerámico con pseudoglifos, encontrado como parte del ajuar funerario del Entierro 1 en esta misma estructura (Figura 22); en él

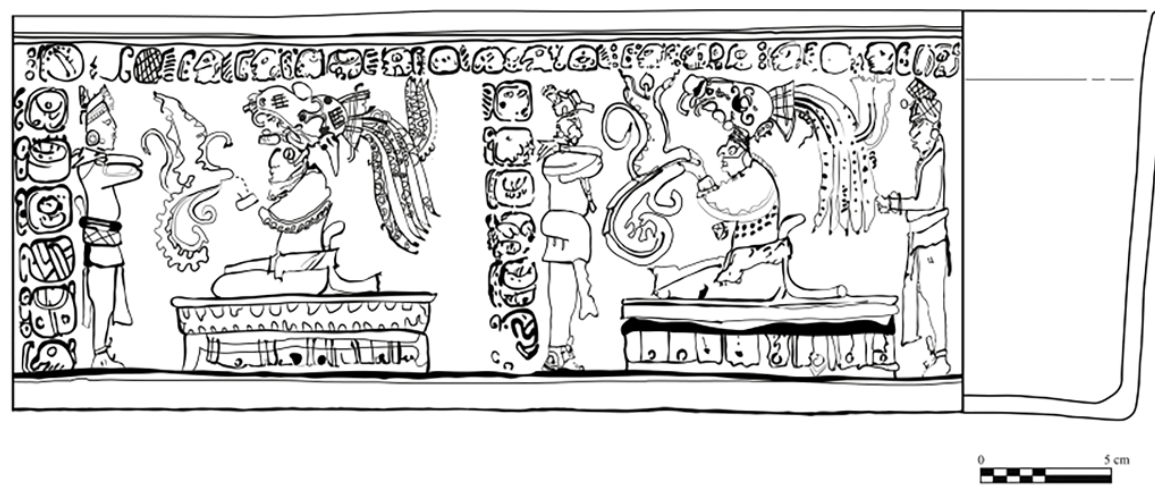

Figura 22. Dibujo de un vaso polícromo, perteneciente al Entierro 1 (Dibujo: Kenichiro Tsukamoto). 


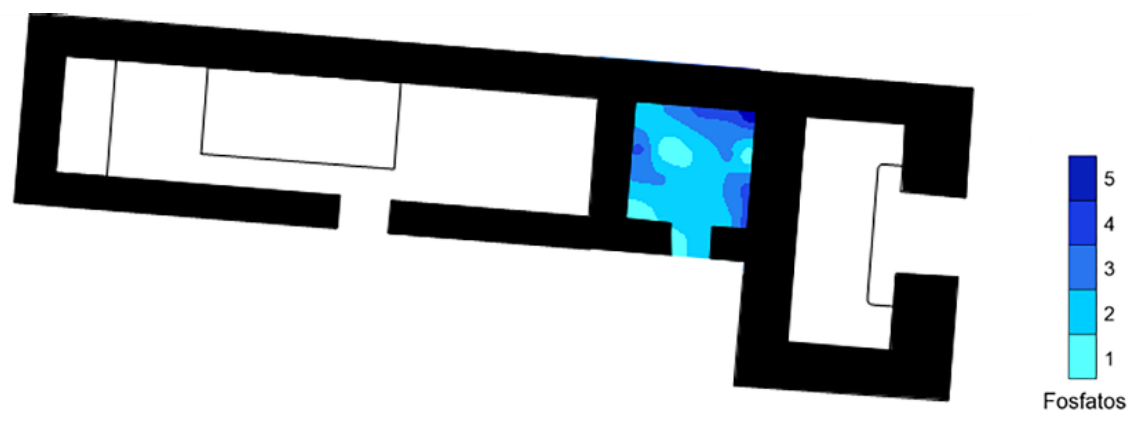

Figura 23. Distribución de fosfatos y carbonatos en la Estructura GZ6 (Dibujo: Xanti Ceballos y Kenichiro Tsukamoto).

se observan rituales de fuego realizados por dos personajes ricamente ataviados con tocados, cada uno en una banqueta diferente, emitiendo lo que parece ser una flama (Tsukamoto et al., 2015: 207).

\section{Estructura GZ6}

En el cuarto muestreado de la estructura, los niveles de fosfatos están agrupados en las orillas norte, oriente y poniente, mientras que en el centro se encuentran niveles muy bajos del mismo (Figura 23); en el caso de los carbonatos, se presentan en niveles altos, a excepción de las orillas poniente y oriente, donde están los niveles más bajos, en los mismos lugares que los fosfatos antes mencionados (Figura 24).

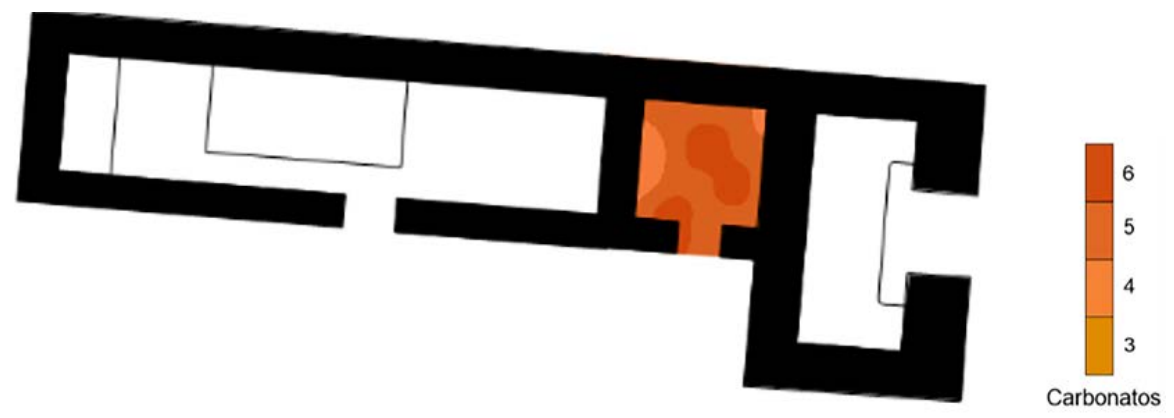

Figura 24. Distribución de fosfatos y carbonatos en la Estructura GZ6 (Dibujo: Xanti Ceballos y Kenichiro Tsukamoto). 
Los valores más altos de residuos proteicos se encuentran acumulados en forma de bandas en las orillas oriente y poniente del cuarto, dejando prácticamente un pasillo sin rastro de proteínas al centro de ellas (Figura 25). Las dimensiones del cuarto son demasiado pequeñas, de $2 \times 2 \mathrm{~m}$, por lo que podría tratarse de un lugar destinado al almacenamiento de bienes, en particular de alimentos de origen animal.

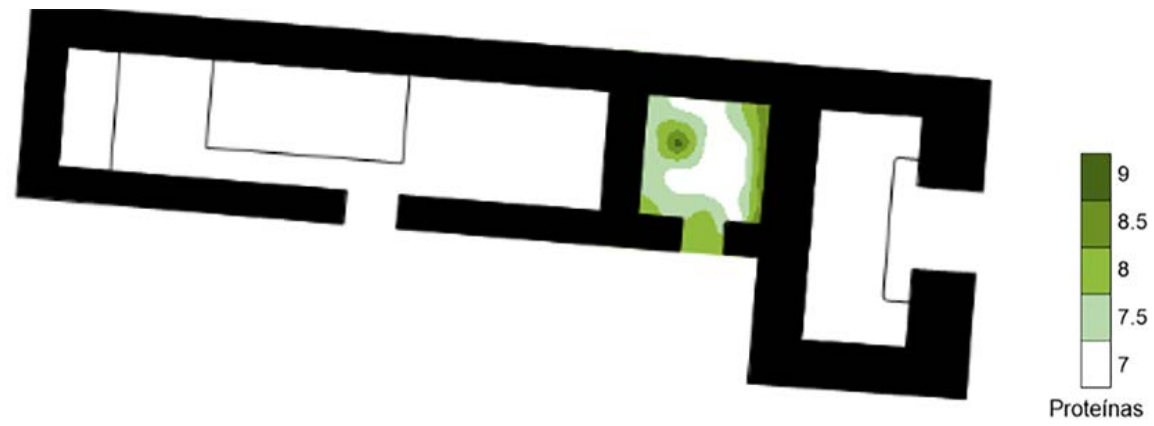

Figura 25. Distribución de proteínas en la Estructura GZ6

(Dibujo: Xanti Ceballos y Kenichiro Tsukamoto).

Los ácidos grasos se agrupan en los costados y al norte, alejados de la entrada al cuarto, al igual que las proteínas (Figura 26). Sus valores son intermedios, concentrándose en una de las esquinas; no obstante, la conjunción de proteínas y ácidos grasos, así como la distribución en las orillas de los residuos químicos, puede ser la confirmación de que el cuarto fue un área de almacenamiento de bienes vegetales o animales.
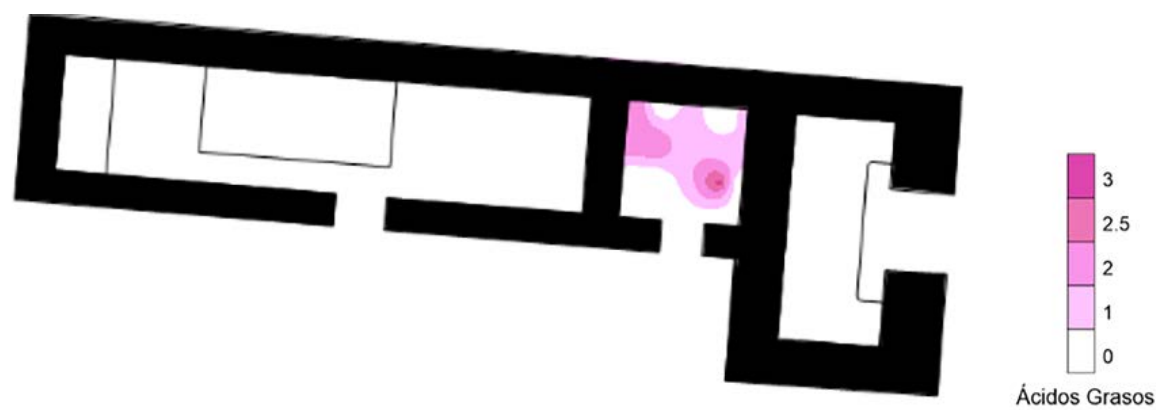

Figura 26. Distribución de ácidos grasos

en la Estructura GZ6 (Dibujo: Xanti Ceballos y Kenichiro Tsukamoto). 
El pH del cuarto presenta valores altos, lo cual, al igual que en el caso de la Estructura GZ1, puede estar relacionado con la ceniza que se encontró como parte del ritual de terminación (Figura 27). Asimismo, la excavación detectó una mancha negra en la esquina noreste, donde los valores de $\mathrm{pH}$ son más altos, quizá producto de la quema del piso durante el mismo ritual (Tsukamoto y López Camacho, 2011: 146). Los carbohidratos, al igual que los otros residuos, muestran un patrón de enriquecimiento en las orillas, con las concentraciones más altas al norte y oriente del cuarto (Figura 28).

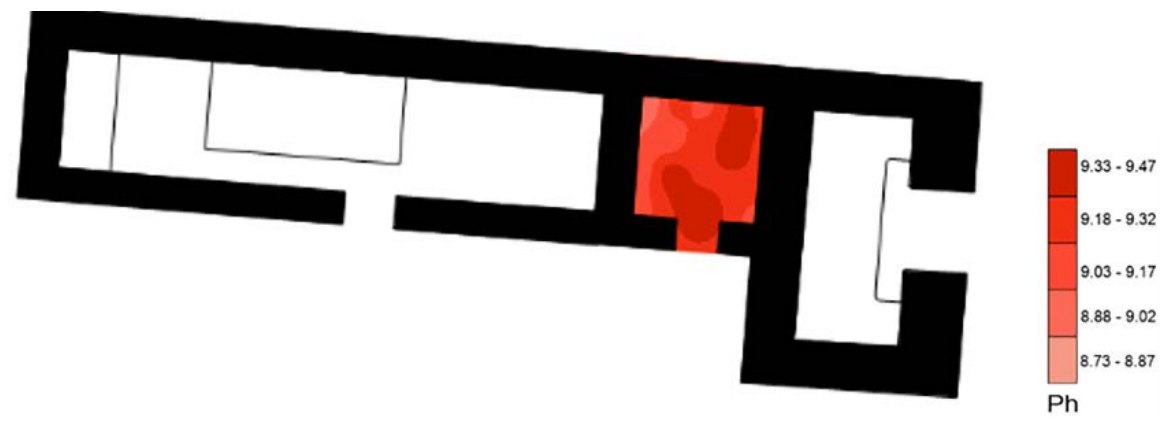

Figura 27. Distribución de pH en la Estructura GZ6

(Dibujo: Xanti Ceballos y Kenichiro Tsukamoto).

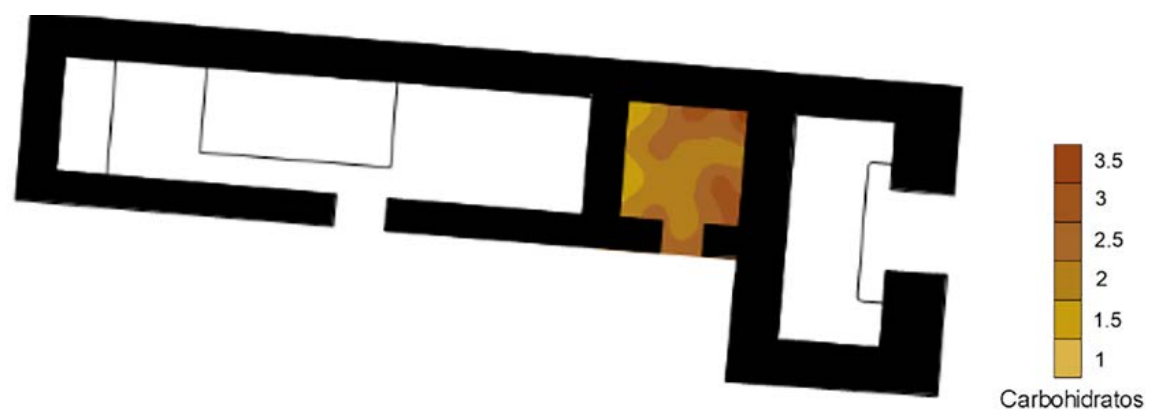

Figura 28. Distribución de carbohidratos en la Estructura GZ6

(Dibujo: Xanti Ceballos y Kenichiro Tsukamoto). 
Planos comparativos entre estructuras

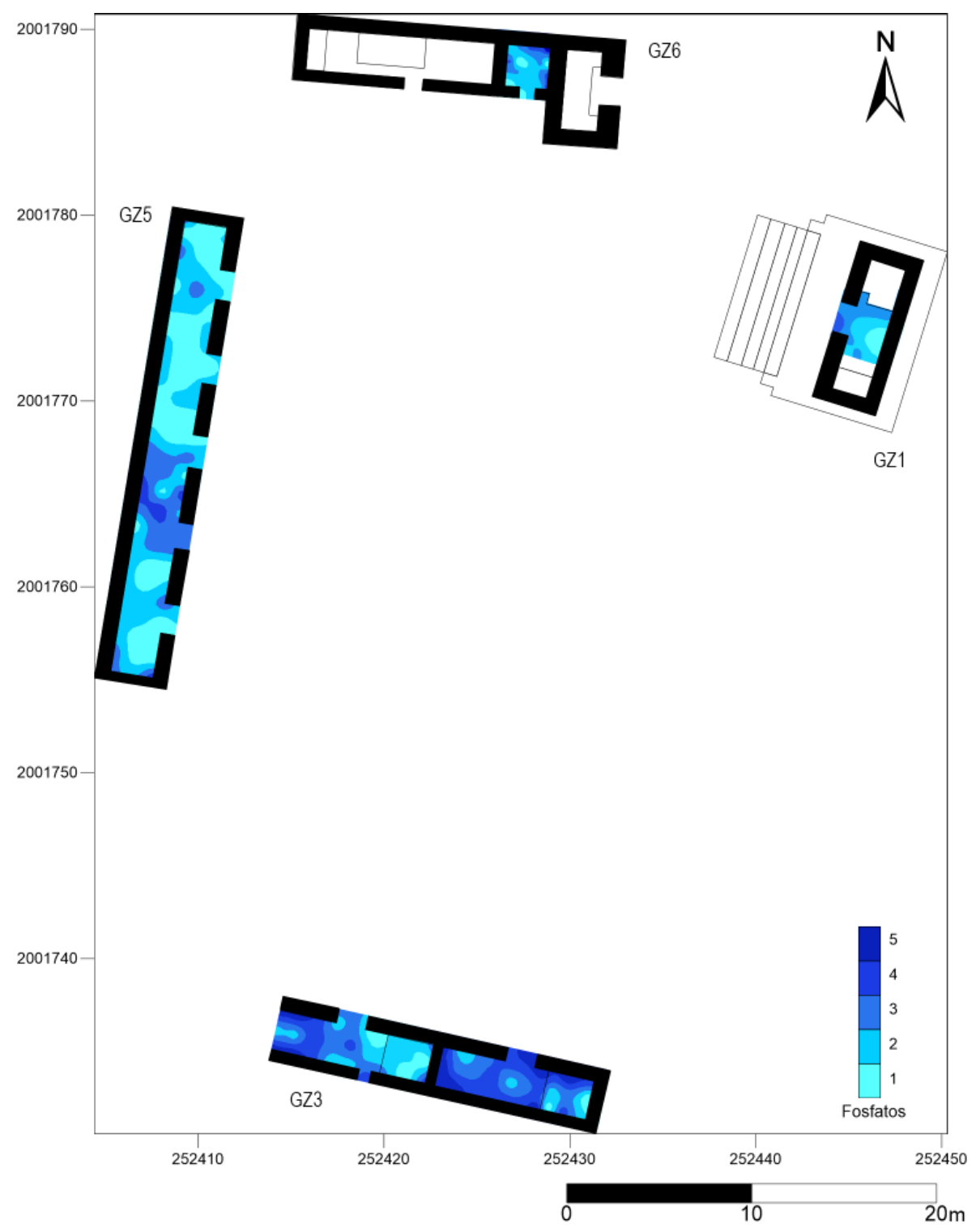

Figura 29. Plano de distribución de fosfatos en el Grupo Guzmán (Dibujo: Xanti Ceballos y Kenichiro Tsukamoto). 


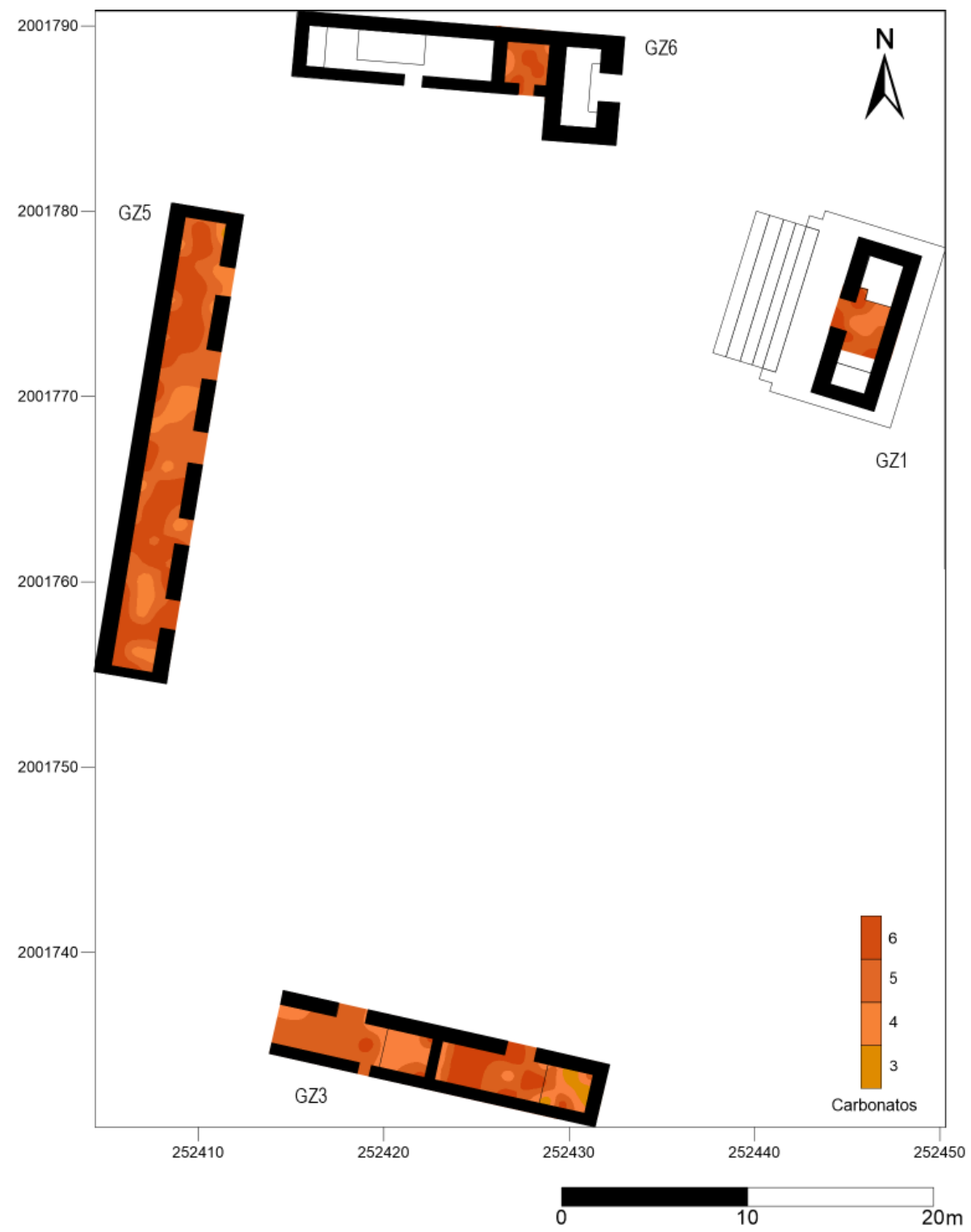

Figura 30. Plano de distribución de carbonatos en el Grupo Guzmán (Dibujo: Xanti Ceballos y Kenichiro Tsukamoto). 


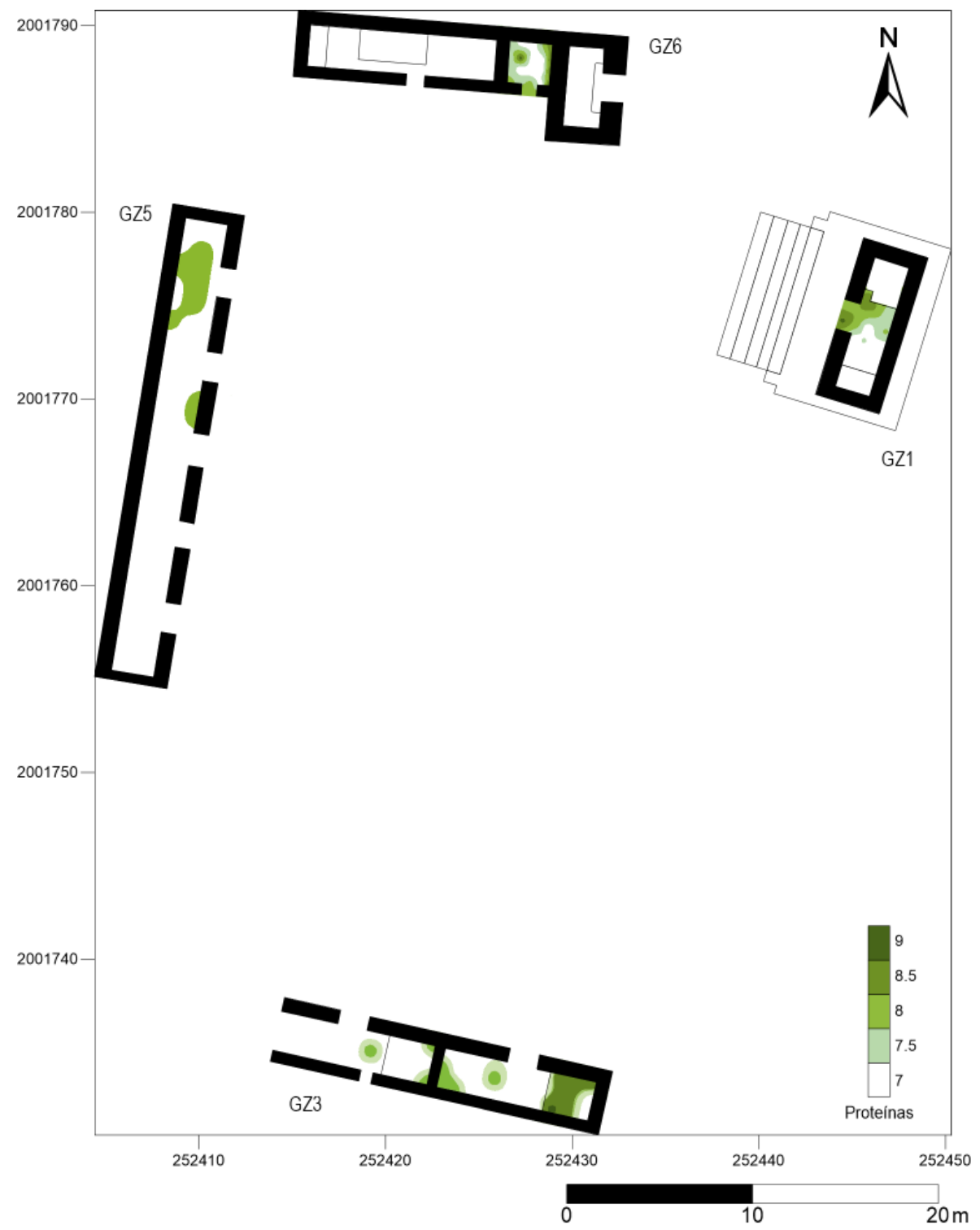

Figura 31. Plano de distribución de proteínas en el Grupo Guzmán (Dibujo: Xanti Ceballos y Kenichiro Tsukamoto). 


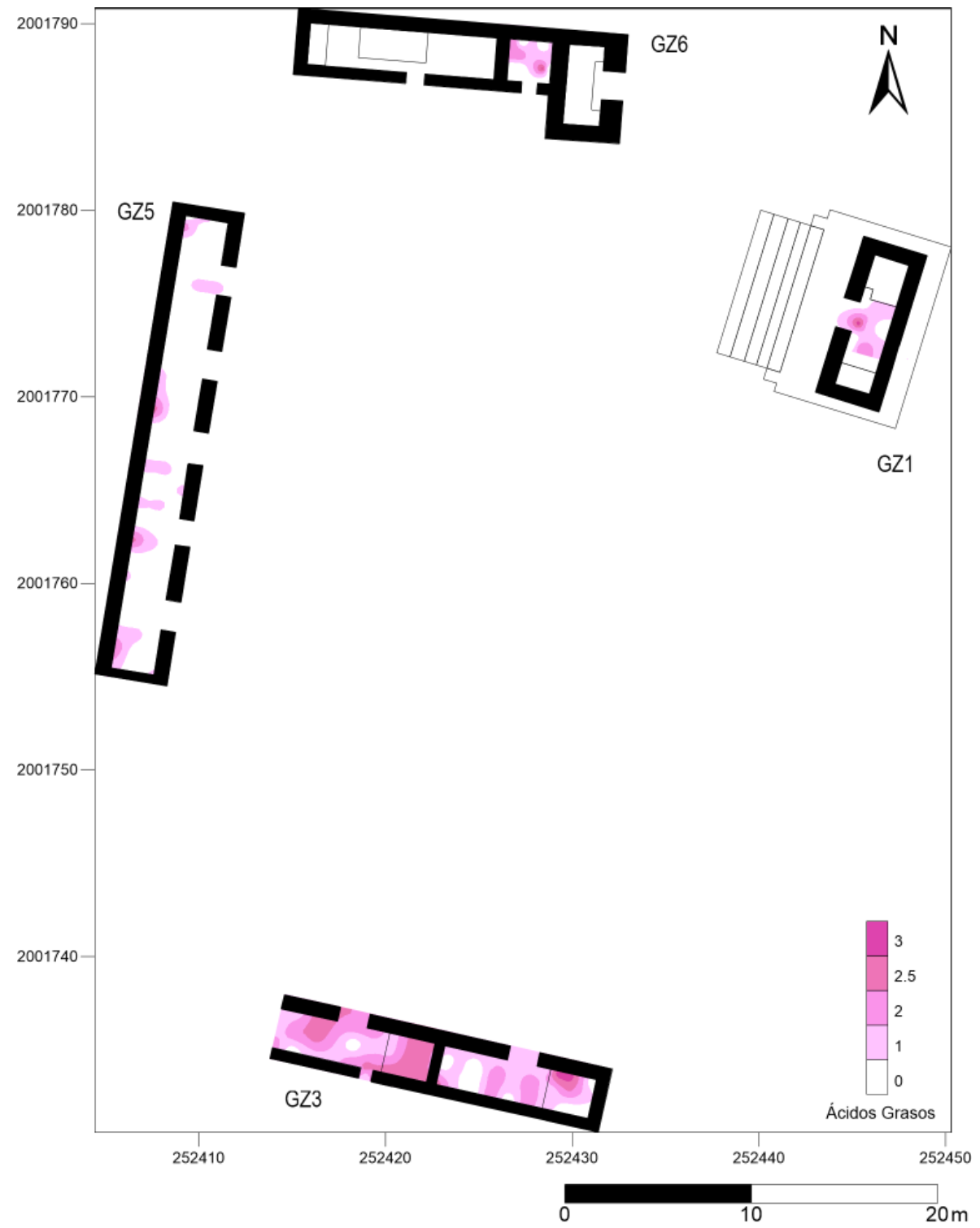

Figura 32. Plano de distribución de ácidos grasos en el Grupo Guzmán (Dibujo: Xanti Ceballos y Kenichiro Tsukamoto). 


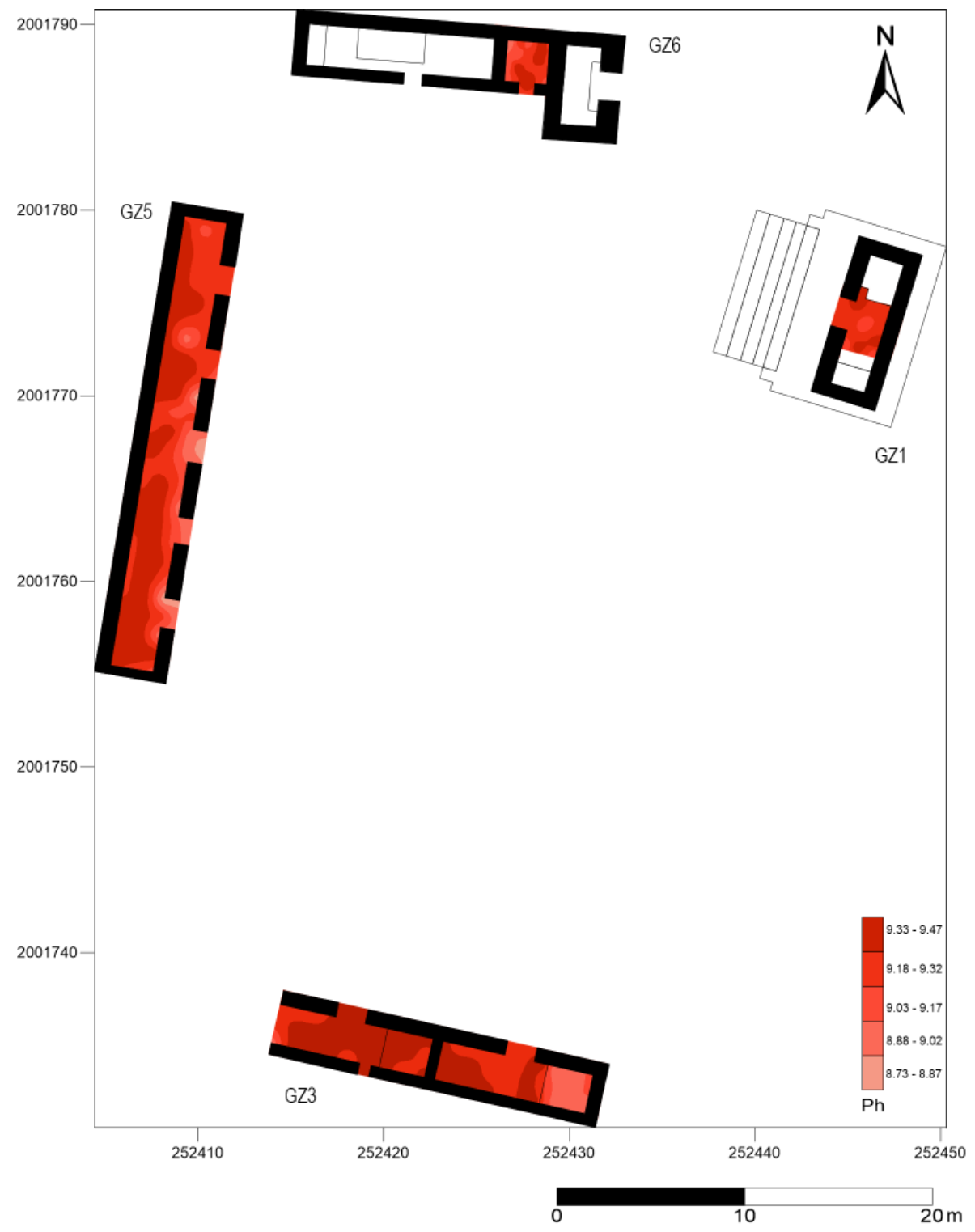

Figura 33. Plano de distribución de pH en el Grupo Guzmán (Dibujo: Xanti Ceballos y Kenichiro Tsukamoto). 


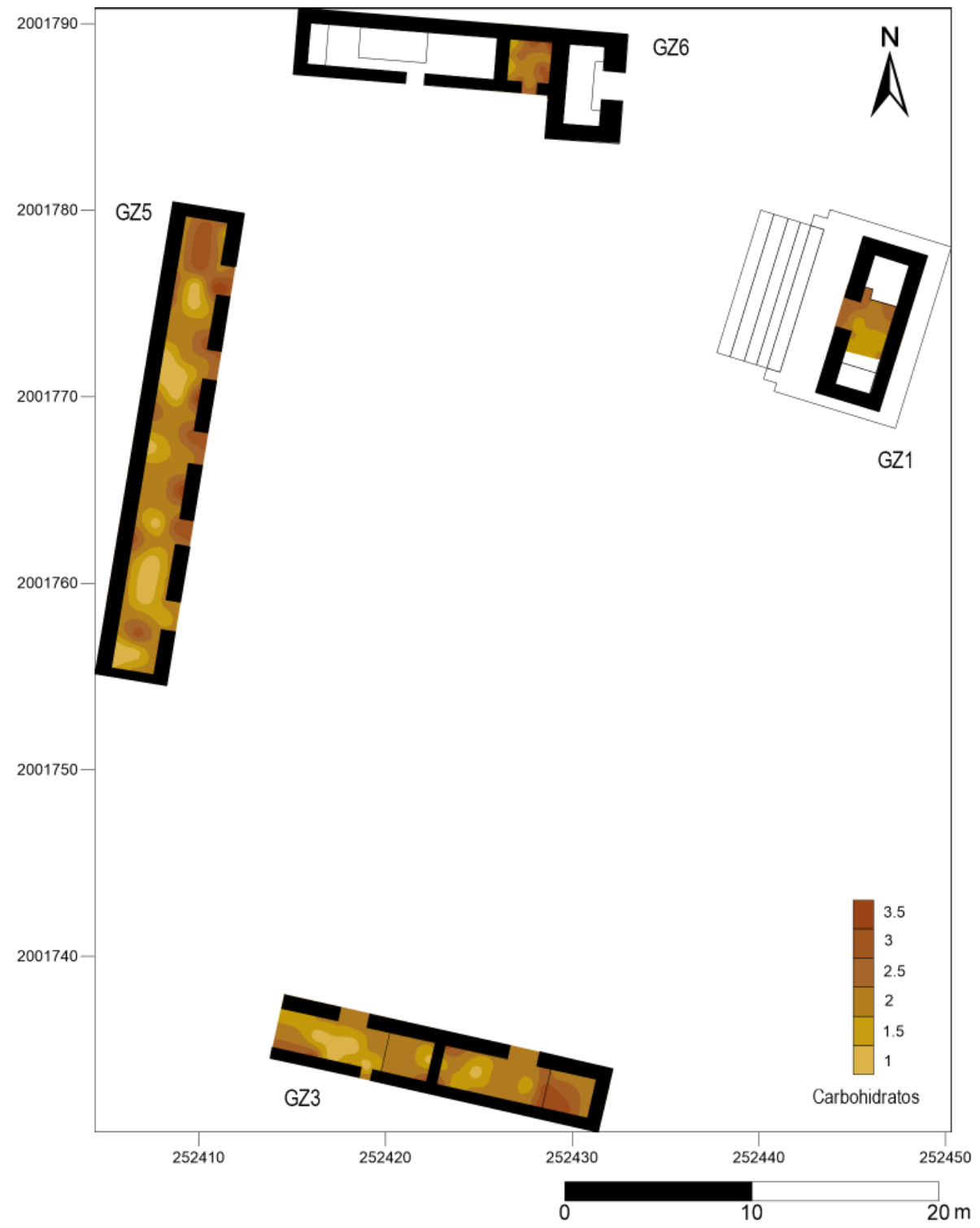

Figura 34. Plano de distribución de carbohidratos en el Grupo Guzmán (Dibujo: Xanti Ceballos y Kenichiro Tsukamoto). 


\section{Discusión: el análisis comparativo de diferentes actividades en las estructuras inferidas a través de los residuos químicos}

Los análisis de residuos químicos sirven como una herramienta que permite verificar la hipótesis sobre la función de las estructuras del Grupo Guzmán. Gracias a ellos se pueden proponer, de una manera sencilla y relativamente rápida, las áreas de actividad de las mismas, comparar resultados y ampliar las interpretaciones sobre su función. La información de las áreas de actividad de las estructuras GZ3 y GZ5, junto con los muestreos parciales en las estructuras GZ1 y GZ6, permiten saber que el Grupo Guzmán es una unidad habitacional de tipo plazuela, donde cada estructura tiene funciones diferentes pero complementarias (Chase y Chase, 2014).

La organización espacial de las estructuras es similar a las unidades residenciales excavadas en Aguateca, Guatemala, donde los trabajos arqueológicos develaron la presencia de varias áreas de actividad dentro de una estructura. La diferencia sería que en Aguateca las áreas de actividad fueron identificadas mediante los objetos dejados in situ debido al abandono súbito que presentaron mientras que, en el caso de las estructuras del Grupo Guzmán, estas áreas de actividad fueron reconstruidas mediante el análisis de residuos químicos. Asimismo, la arquitectura y ensambles cerámicos fueron similares a los encontrados en las residencias de Aguateca. Es importante mencionar que, aunque muchas de las actividades cotidianas fueron reconstruidas mediante el análisis de los residuos químicos debido al abandono gradual de las estructuras, no existió evidencia directa de áreas de actividad exclusivas del género masculino o femenino, como ensambles de figurillas, malacates o juegos de herramientas para talla en piedra, como las que fueron halladas en Aguateca (Triadan, 2007). Por lo tanto, la reconstrucción de las actividades no llega a ese nivel de detalle.

Las áreas de actividad de la Estructura GZ3 permiten afirmar su función residencial, debido a los patrones de enriquecimiento que muestran tanto los cuartos como las banquetas, lo cual no descarta que en ella también se llevaran a cabo actividades públicas, como la recepción de visitas. El alto contenido de todos los residuos, a excepción de las proteínas - localizadas sólo en las esquinas y el centro de los cuartos, así como en la banqueta oriente-, resulta característico de una casa y da cuenta de actividades cotidianas como comer, dormir, preparar y almacenar alimentos. Asimismo, los valores encontrados en las banquetas muestran que fueron bastante utilizadas al igual que los cuartos. Los altos valores de $\mathrm{pH}$ en el cuarto poniente parecen ser consistentes con las áreas que fueron quemadas durante el ritual de terminación y en donde se recolectaron algunos carbones.

Las áreas de actividad de la Estructura GZ5 son diferentes a las otras estructuras del Grupo Guzmán. Las concentraciones de residuos son bajas en general y en el caso de las proteínas y los ácidos grasos se encuentran aislados, en lugares específicos como las orillas y al norte de la estructura, lo que implicaría que en 
este espacio se llevaron a cabo actividades esporádicas o poco contaminantes, que descartan la idea de que se trate de una residencia. La arquitectura de galería corrida, con alta visibilidad desde la plaza, y los resultados del análisis de residuos refuerzan la idea de que podría tratarse de un edificio administrativo, lo cual dejó una huella química leve en los pisos.

Las áreas de actividad en la Estructura GZ1 permiten afirmar que su función como templo incluyó rituales, en los que se involucraron fluidos ricos en proteínas y ácidos grasos, posiblemente provenientes de sangre y resinas, sin descartar el derramamiento de bebidas; la mayor parte de estos rituales se llevaron a cabo en la entrada del templo. Lo anterior es consistente con la ampliación de la escalinata jeroglífica para llevar a cabo espectáculos en ella, donde la entrada pudo tener un papel fundamental en el ritual público, con una buena visibilidad desde la plaza (Tsukamoto, 2014: 62).

En el caso de la Estructura GZ6, el agrupamiento de los residuos en las orillas del cuarto central hace pensar que fue una habitación destinada al almacenamiento de alimentos, como se había propuesto anteriormente (Tsukamoto et al., 2015: 210). Debido al tamaño, junto con los enriquecimientos de ácidos grasos y residuos proteicos, inicialmente se creyó que podría ser un temazcal; sin embargo, la falta de elementos como banquetas laterales o las piedras para generar calor que se han reportado en otros sitios mayas, no corroboraron esta hipótesis. Los patrones de $\mathrm{pH}$ muestran una concentración alta en la entrada y en la esquina noreste de la estructura, por lo que no hay algún círculo específico central donde se pudiese afirmar que hubo un fogón que dejara alguna huella química (Chase y Chase, 2014: 11). Es importante mencionar que estas dos últimas estructuras ofrecen una información sesgada y no ha sido posible profundizar más sobre su función debido a que no fueron muestreadas en su totalidad.

Cuando las estructuras GZ1 y GZ6 fueron excavadas, se registraron materiales pertenecientes a un ritual de terminación, los cuales fueron estudiados por Tsukamoto mediante un análisis espacial. Él llegó a la conclusión que tanto GZ1 como GZ6 formaron parte de un ritual de terminación reverencial, debido a que los objetos líticos y cerámicos fueron intencionalmente depositados en cada espacio, con algún significado especial para los habitantes, pero sin la destrucción intencional de los enemigos (Tsukamoto, 2017: 1643).

Relacionando esta información con la distribución de los análisis de residuos, una explicación plausible, en este caso particular, sería que los artefactos depositados durante el ritual de terminación pudieron ser colocados sobre los pisos de las estructuras donde se utilizaron; de ahí que la Estructura GZ3 tenga cerámica y poca lítica, GZ5 tenga más lítica que cerámica, GZ1 alguna lítica y cerámica, y el cuarto central de GZ6 una concentración de cerámica al norte, en la cual predominan los fragmentos de olla, con algunos cuencos y platos, sin incluir elementos líticos, como lascas, excéntricos, bifaciales, manos y metates, que sí se encontraron en los otros dos cuartos de la misma estructura (Tsukamoto, 2017: 1640). 
La presencia de carbohidratos en todas las estructuras, independientemente de su función, podría deberse al derramamiento de las bebidas servidas durante los festejos de los lakamob, probablemente líquidos a base de maíz o cacao, o quizá el balché, una bebida fermentada embriagante de la corteza del árbol con el mismo nombre, mezclado con miel, cuyo uso se ha documentado desde las épocas de la conquista en Yucatán y que se preserva como bebida ritual entre los mayas peninsulares y los lacandones hoy en día (Gabriel, 2007; Pugh, 2009). Actualmente, esta bebida se utiliza como una ofrenda que se presenta en el altar, así como para la aspersión a los cuatro rumbos en la entrega final de las ofrendas y como parte de los alimentos que se consumen en las ceremonias (Gabriel, 2007: 158).

\section{Conclusión}

Las estructuras del Grupo Guzmán fueron utilizadas intensamente por sus habitantes, lo cual se ve reflejado en sus áreas de actividad. Altas concentraciones de residuos fueron encontradas en las Estructuras GZ1, GZ3 y GZ6, mientras que la Estructura GZ5 muestra enriquecimientos bajos en su galería; sin embargo, es interesante notar que la concentración de carbohidratos está presente en todas ellas y puede estar relacionada con las bebidas ofrecidas en las frecuentes fiestas realizadas por los lakamob, en las que participaron los habitantes del Grupo Guzmán así como personas externas a éste, de acuerdo con el tamaño de aforo de la plaza (Tsukamoto, 2014: 57). Sin embargo, estudios más específicos, como la cromatografía de gases acoplada a espectrometría de masas, serán necesarios para corroborar esta afirmación.

\section{Agradecimientos}

Externamos nuestro agradecimiento a los participantes del Proyecto Arqueológico El Palmar, especialmente al codirector del proyecto, Javier López Camacho, profesor de la Escuela Nacional de Antropología e Historia, por su invaluable apoyo académico. Asimismo agradecemos al Consejo de Arqueología del Instituto Nacional de Antropología e Historia por los permisos otorgados para su realización y a la delegada del Centro INAH Campeche, Adriana Velázquez Morlet, por las facilidades brindadas para llevarlo a cabo. Este estudio contó con el apoyo constante de los pobladores del Ejido Kiché Las Pailas y fue financiado por la JSPS Postdoctoral Research Fellowship (15J00280) y por la JSPS KAKENHI (19K13408; 21402008), a través de la Universidad de Estudios Extranjeros de Kioto, la Universidad Yamagata, así como el financiamiento inicial de la Universidad de California, Riverside, concedidos a Kenichiro Tsukamoto. Finalmente agradecemos a los dos revisores por sus atinados comentarios, que mejoraron considerablemente este trabajo. 


\section{Bibliografía}

Ashmore, Wendy y Richard Wilk

1988 "Household and Community in the Mesoamerican Past", Household and Community in the Mesoamerican Past, pp. 1-13, R. Wilk y W. Ashmore (eds.). Albuquerque, Nuevo México: University of New Mexico Press.

Barba, Luis A.

1986 "La química en el estudio de las áreas de actividad”, Unidades habitacionales de Mesoamérica y sus áreas de actividad, pp. 21-39, Linda Manzanilla (ed.). México: Universidad Nacional Autónoma de México.

1990 "El uso del análisis de fosfato en sitios arqueológicos de Xochipala, Guerrero", Apéndice en Arqueología de Xochipala, pp. 257-272, Paul Schmidt (ed.). México: Universidad Nacional Autónoma de México.

2007 "Chemical Residues in Lime-Plastered Archaeological Floors", Geoarchaeology, 22 (3): 439-453. DoI: https://doi.org/10.1002/gea.20160.

Barba, Luis y Gregorio Bello

1978 "Análisis de fosfato en el piso de una casa habitación actual", Notas Antropológicas, 24 (1): 188-193.

Barba, Luis y Linda Manzanilla

1987 "Estudio de áreas de actividad", Cobá, Quintana Roo. Análisis de dos unidades habitacionales mayas del horizonte Clásico, pp. 69-115, Linda Manzanilla (ed.). México: Universidad Nacional Autónoma de México.

Barba, Luis, Roberto Rodríguez y José Luis Córdova

1991 Manual de técnicas microquímicas de campo para la arqueología. México: Universidad Nacional Autónoma de México, Instituto de Investigaciones Antropológicas.

Barba, Luis y Agustín Ortiz

1992 "Análisis químico de pisos de ocupación: un caso etnográfico en Tlaxcala, México”, Latin American Antiquity, 3 (1): 63-82. Dol: https://doi. org/10.2307/971930.

Barba Luis, Luz Lazos, Agustín Ortiz, Karl Link y Leonardo López Luján

1996 "The Chemical Analysis of Residues in Floors and the Reconstruction of Ritual Activities at the Templo Mayor, Mexico", Archaeological Chemistry: Organic, Inorganic and Biochemical Analysis, pp. 139-156, Mary V. Orna (ed.). Washington: Chemical Society of America.

Barba Luis, Agustín Ortiz, Karl Link, Luz Lazos y Leonardo López Luján

1997 "La arquitectura sagrada y sus usos. Estudio geofísico y químico de la Casa de las Águilas, Tenochtitlan, México”, Revista de Arqueología, 198: 44-53.

Barba Luis, Luz Lazos, Karl Link, Agustín Ortiz y Leonardo López Luján

1998 “La arqueometría en la Casa de las Águilas”, Arqueología Mexicana, 31: 20-27. 
Becker, Marshall

1991 "Plaza Plans at Tikal, Guatemala, and at Other Lowland Maya Sites: Evidence for Patterns of Cultural Change", Cuadernos de Arquitectura Mesoamericana, 14: 11-26.

Ceballos Pesina, Xanti Sirani

2020 "Actividades en las unidades habitacionales mayas durante el periodo Clásico (250-850 d.C.): el caso de la Estructura GZ3 del Grupo Guzmán en El Palmar, Campeche, México", tesis de licenciatura en Arqueología. México: Escuela Nacional de Antropología e Historia.

Chase, Diane Z. y Arlen F. Chase

2014 "Ancient Maya Houses, Households, and Residential Groups at Caracol, Belize", Belizean Archaeology, 11: 3-17.

Gabriel, Marianne

2007 "El uso ritual de alcohol, tabaco, cacao e incienso en las ceremonias agrarias de los mayas yucatecos contemporáneos", Estudios de Cultura Maya, XXIX: 155-184. DoI: https://doi.org/10.19130/iifl.ecm.2007.29.115.

Hayden, Brian D. y Aubrey Cannon

1983 "Where the Garbage Goes: Refuse Disposal in the Maya Highlands", Journal of Anthropological Archaeology, 2 (2): 117-163. Dol: https://doi.org/10.1016/02784165(83)90010-7.

Hutson, Scott R., Travis W. Stanton, Aline Magnoni, Richard Terry y Jason Craner

2007 "Beyond the Buildings: Formation Processes of Ancient Maya Houselots and Methods for the Study of Non-Architectural Space", Journal of Anthropological Archaeology, 26 (3): 442-473. DoI: https://doi.org/10.1016/j.jaa.2006.12.001.

Lacadena García-Gallo, Alfonso

2008 "El título lakam: evidencia epigráfica sobre la organización tributaria y militar interna de los reinos mayas del Clásico”, Mayab, 20: 23-43.

Manzanilla, Linda y Luis Barba

1990 "The Study of Activities in Classic Households: Two Case Studies from Coba and Teotihuacan", Ancient Mesoamerica, 1 (1): 41-49. Dor: https://doi. org/10.1017/S0956536100000067.

Mejía Pérez Campos, Elizabeth y Luis Barba Pingarrón

1988 "El análisis de fosfatos en la arqueología. Historia y perspectivas", Anales de Antropología, 25 (1): 128-147. Dol: http://dx.doi.org/10.22201/ iia.24486221e.1988.1.15798.

Ortiz, Agustín y Luis Barba

1992 "Estudio químico de los pisos del Satunsat en Oxkintok, Yucatán”, Oxkintok, 4: 119-126. Madrid: Misión Arqueológica de España en México. 
Ortiz, Agustín, Luis Barba y Alessandra Pecci

2017 "Lo tangible de lo intangible: los residuos químicos como medio para estudiar las actividades rituales del pasado", Las ciencias aplicadas al estudio del patrimonio cultural, pp. 223-260, Agustín Ortiz Butrón (ed.). México: Red de Ciencias Aplicadas a la Investigación y Conservación del Patrimonio Cultural, Universidad Nacional Autónoma de México, Instituto de Investigaciones Antropológicas.

Pecci, Alessandra, Agutín Ortiz y Luis Barba

2017 "Los residuos químicos de la producción de pulque. Etnoarqueometría y arqueología experimental”, Anales de Antropología, 51 (1): 39-55. DoI: https:// dx.doi.org/10.1016/j.antro.2016.11.002.

Pugh, Timothy W.

2009 "Cacao, Gender, and the Northern Lacandon God House", Chocolate in Mesoamerica: A Cultural History of Cacao, pp. 367-383, Cameron McNeil (ed.). Florida: University Press of Florida.

Stuart, David

1988 "The Rio Azul Cacao Pot: Epigraphic Observations on the Function of Maya Ceramic Vessels", Antiquity, 62 (234): 153-157. Dol: https://doi.org/10.1017/ S0003598X00073634.

2010 "The LAKAM Sign", nota publicada en Maya Decipherment. Disponible en: $<$ https://decipherment.wordpress.com/2010/03/08/the-lakam-logogram/> [consultada el 10 de marzo de 2018].

Triadan, Daniela

2000 "Elite Household Subsistence at Aguateca, Guatemala", Mayab, 13: 46-56.

2007 "Warriors, Nobles, Commoners and Beasts: Figurines from Elite Buildings at Aguateca, Guatemala”, Latin American Antiquity, 18 (3): 269-293. Dol: https:// doi.org/10.2307/25478181.

Tsukamoto, Kenichiro

2014 "Multiple Identities on the Plazas: The Classic Maya Center of El Palmar, Mexico", Mesoamerican Plazas: Arenas of Community and Power, pp. 50-67, Kenichiro Tsukamoto y Takeshi Inomata (eds.). Tucson: University of Arizona Press.

2017 "Reverential Abandonment: A Termination Ritual at the Ancient Maya Polity of El Palmar", Antiquity, 91: 1630-1646. Dol: https://doi.org/10.15184/ aqy.2017.143.

Tsukamoto, Kenichiro y Javier López Camacho (eds.)

2011 "Proyecto Arqueológico El Palmar: urbanización del espacio social en las tierras bajas mayas. Informe de la tercera temporada de campo 2010-2011". México: Archivo Técnico del Instituto Nacional de Antropología e Historia.

2018 "Proyecto Arqueológico El Palmar: urbanización del espacio social en las tierras bajas mayas. Informe de la sexta temporada de campo 2016". México: Archivo Técnico del Instituto Nacional de Antropología e Historia. 
Tsukamoto, Kenichiro, Javier López Camacho, Luz Evelia Campaña, Hirokazu Kotegawa y Octavio Esparza

2015 "Political Interactions among Social Actors: Spatial Organization at the Classic Maya Polity of El Palmar, Campeche, Mexico", Latin American Antiquity, 26 (2): 200-220. Dol: https://doi.org/10.7183/1045-6635.26.2.200.

Tsukamoto, Kenichiro y Octavio Esparza

2016 "Ajpach' Waal: The Hieroglyphic Stairway at the Guzmán Group of El Palmar, Campeche, Mexico", Maya Archaeology, 3: 30-55, C. Golden, S. Houston y J. Skidmore (eds.). San Francisco: Pre-Columbia Mesoweb Press.

Tsukamoto, Kenichiro, Luz Evelia Campaña, Xanti Sirani Ceballos, Renato José Zamudio, Dulce María Ramírez, Melina García Hernández, Hirokazu Kotegawa y Javier López Camacho 2017 "Proyecto Arqueológico El Palmar en Campeche, México: resultados de la temporada de campo 2016", XXXI Simposio de Investigaciones Arqueológicas en Guatemala, pp. 431- 440. Guatemala: Ministerio de Cultura y Deportes, Dirección General del Patrimonio Cultural y Natural y Asociación Tikal.

Xanti Sirani Ceballos Pesina. Mexicana. Licenciada en Arqueología por la Escuela Nacional de Antropología e Historia, México. Su interés de investigación se centra en la desigualdad de las sociedades complejas tempranas a través del estudio de las unidades habitacionales, la cerámica y la arqueometría. Es colaboradora en el Proyecto Arqueológico El Palmar: urbanización del espacio social en las tierras bajas mayas. Entre sus publicaciones recientes se encuentra "Proyecto Arqueológico El Palmar en Campeche, México: resultados de la temporada de campo 2016", en coautoría.

xanti_sirani_1@hotmail.com

Agustín Ortiz Butrón. Mexicano. Licenciado en Arqueología por la Escuela Nacional de Antropología e Historia, maestro y doctor en Antropología por el Programa de Posgrado en Antropología de la Universidad Nacional Autónoma de México. Es el encargado del Laboratorio de Prospección Arqueológica del Instituto de Investigaciones Antropológicas, unam, en la sección de estudios de residuos químicos en materiales arqueológicos y su principal línea de investigación se inserta en el campo de la arqueometría, que abarca la aplicación de técnicas de fotografía aérea, prospección geofísica y estudio de residuos químicos en arqueología. Entre sus publicaciones recientes se encuentran "Los residuos químicos. Indicadores arqueológicos para entender la producción, preparación, consumo y almacenamiento de alimentos", "Los residuos químicos de la producción de pulque", "Residuos químicos y el contexto de enterramiento" y "Lo tangible de lo intangible: los residuos químicos como medio para estudiar las actividades rituales del pasado", todas en coautoría.

ortizbutron@gmail.com 
Luis Barba Pingarrón. Mexicano. Ingeniero Químico Industrial por el Instituto Politécnico Nacional; maestro en Ciencias (Geología Arqueológica) por la Universidad de Georgia y doctor en Antropología por la Facultad de Filosofía y Letras de la Universidad Nacional Autónoma de México. Tiene a su cargo el Laboratorio de Prospección Arqueológica del Instituto de Investigaciones Antropológicas, unam. Sus principales líneas de investigación son la arqueometría, las técnicas de prospección arqueológica, los residuos químicos en pisos y cerámicas y la tecnología prehispánica y colonial. Entre sus publicaciones más recientes se encuentran "Los residuos químicos de la producción de pulque", "Cacao Residues in Vessels from Chocolá, an Early Maya Polity in the Southern Guatemalan Piedmont, Determined by Semi-Quantitative Testing and High-Performance Liquid Chromatography" y "Chemical Residues as Anthropic Activity Markers. Ethnoarchaeology, Experimental Archaeology and Archaeology of Food Production and Cosumption”, todas en coautoría.

barba@unam.mx

Araceli Vázquez Villegas. Mexicana. Pasante de Arqueología por la Escuela Nacional de Antropología e Historia. Su principal línea de investigación es el patrón de asentamiento y la cerámica del sur de Quintana Roo. Es colaboradora de los proyectos "Prácticas de prospección arqueológica en el sur de Quintana Roo" y "El Palmar: urbanización del espacio social en las tierras bajas mayas". Entre sus publicaciones recientes se encuentran "Ensayo preliminar del análisis de restos óseos animales y humanos del sitio Nueva Esperanza, Quintana Roo", "Lakin Kah Las Palmas" y "Noh Kah: An Archaeological Site in Extreme Southeastern Quintana Roo", todas en coautoría.

araceli_vazquez15@hotmail.com

Kenichiro Tsukamoto. Japonés. Licenciado en Arqueología por la Escuela Nacional de Antropología e Historia y doctor en Antropología por la Universidad de Arizona, Estados Unidos. Adscrito al Departamento de Antropología de la Universidad de California, Riverside. Su investigación se centra en la interacción entre las relaciones sociales y las prácticas reflejadas en los entornos espaciales y materiales de las sociedades complejas tempranas. Es director del Proyecto Arqueológico El Palmar: urbanización del espacio social en las tierras bajas mayas. Entre sus publicaciones más recientes se encuentran "Ajpach' Waal: The Hieroglyphic Stairway at the Guzmán Group of El Palmar, Campeche, Mexico", The Past in the Present: An Introduction to Archaeology y "Reverential Abandonment: A Termination Ritual at the Ancient Maya Polity of El Palmar", esta última como autor único.

kenichiro.tsukamoto@ucr.edu 